\title{
SUR LA FAMILLE DES PARAPAGURIDAE SMITH, 1882. DESCRIPTION DE TYPHLOPAGURUS FORESTI GEN. NOV., SP. NOV., ET DE QUINZE ESPÈCES OU SOUS-ESPÈCES NOUVELLES DE PARAPAGURUS SMITH (CRUSTACEA, DECAPODA)
}

\author{
par \\ MICHELE DE SAINT LAURENT \\ Muséum national d'Histoire naturelle, Paris, France
}

\section{RESUME}

Ce travail comporte la diagnose de la famille des Parapaguridae Smith, 1882, abandonnée depuis de nombreuses années et rétablie ici sur de nouvelles bases morphologiques, et sa comparaison avec celle des Paguridae Dana. Elle comprend, en plus de Parapagurus Smith, 1879 (= Sympagurus Smith, 1883), les genres Tylaspis Henderson, 1885, Probeebei Boone, 1926 et Typhlopagurus gen. nov., tous trois monospécifiques à ce jour. Une liste complète des espèces de la famille, avec, pour chacune d'elles, les synonymies, les principales références bibliographiques et la distribution, est donnée. Le genre Typhlopagurus est établi pour une forme nouvelle récoltée par l'“Albatross" aux îles Philippines et caractérisée par la réduction et l'armature épineuse des pédoncules oculaires et la disparition totale des cornées; quinze espèces ou sousespèces nouvelles du genre Parapagurus, provenant des récoltes du "Siboga", de la "Galathea" et de 1"Albatross" ou d'autres collections océanographiques, sont décrites.

\section{INTRODUCTION}

Etudiant en 1964 le développement larvaire de plusieurs espèces du genre Parapagurus Smith, nous avions mis en évidence un ensemble de caractères opposant les zoés de ce genre à celles des autres formes de la famille des Paguridae et suggéré que les Parapagurus appartenaient à un groupe taxonomique distinct.

L'étude morphologique des adultes et la révision systématique des espèces du genre Parapagurus ont confirmé notre hypothèse de 1964 : ce genre présente en effet un certain nombre de caractères propres qui le séparent très nettement des représentants de la famille des Paguridae. Nous proposons ici de rétablir la famille des $\mathrm{Pa}-$ rapaguridae Smith, avec le genre Parapagurus Smith comme type, et incluant Tylaspis Henderson, Probeebei Boone et Typhlopagurus gen. nuv. Ceux-ci présentent des particularités qui leur confèrent un aspect notablement différent de celui des Parapagurus, ce qui est probablement en rapport avec leur mode de vie ou leur habitat. Néan- moins des caractères essentiels permettent de grouper les quatre genres dans une même famille.

\section{RAPPEL HISTORIQUE}

La famille des Parapaguridae a été créée par Smith en 1882 pour le genre Parapagurus, qu'il avait établi en 1879. La forme des lamelles branchiales, profondément divisées en deux lobes chez l'espèce-type du genre, $P$. pilosimanus, paraissait alors constituer un caractère unique chez les $\mathrm{Pa}$ gurides, où l'on ne connaissait que des branchies bisériées, à lamelles entières, et avait paru suffisante à cet auteur pour établir une famille distincte.

Le même auteur, en 1883, créait le genre Sympagurus, qu'en dépit de ses affinités évidentes avec Parapagurus il ne classait pas dans les Parapaguridae, à cause de ses lamelles branchiales entières.

Henderson, en 1888, adoptait la famille de Smith : séparant l'ensemble des Pagurides en Lithodea et Pagurodea, il divisait ces derniers en Laminibranchiata et Fibribranchiata : ceux-ci comprenaient la seule famille des Parapaguridae, groupant des formes aussi diverses que Pylocheles spinosus, Paguropsis typicus, Parapagurus pilosimanus et Pagurodes inarmatus, actuellement classées dans quatre familles distinctes.

Dans une courte note de 1891, Bouvier montrait l'hétérogénéité des Fibribranchiata de Henderson et l'affinité de certaines formes pourvues de types branchiaux différents ; il concluait très justement: "les modifications dans la structure des branchies sont... régulièrement progressives et se prêtent mal, par conséquent, aux groupements de la systématique. Les formes à lamelles intermédiaires (Paguristes, Spiropagurus, Sympagurus) rendent ces groupements impossibles ou anormaux. Il n'est pas naturel, par exemple, de 
ranger dans des genres distincts les Parapagurus et les Sympagurus".

Cependant, Ortmann, dans sa classification de 1892, rassemble encore les Parapagurus et les Pylochelidae actuels dans la famille des Parapaguridae.

Après A. Milne Edwards \& Bouvier (1894 et 1900), Alcock, en 1905, inclut définitivement $\mathrm{Pa}$ rapagurus, avec Sympagurus, dans la famille des Paguridae Dana, sous-famille des Eupagurinae Ortmann. Il reconnaît par ailleurs la famille des Pylochelidae.

Ce classement est celui qui a généralement été adopté depuis, notamment par Balss en 1957, dans le chapitre de systématique du volume du Bronns Tierreich consacré aux Décapodes.

En ce qui concerne la synonymie de Sympagurus avec Parapagurus, préconisée par Bouvier en 1891, elle a été adoptée par Balss en 1912, puis par Forest en 1955.

La classification actuelle des Pagurides, modifiant celle de Balss (1957), et proposée par MacDonald, Pike \& Williamson en 1957, suivis par nous mêmes en 1968 (Forest \& de Saint Laurent), reconnaît dans ce groupe deux superfamilles, Coenobitoida d'une part, avec les familles des Pylochelidae, Lomisidae, Coenobitidae et Diogenidae, et Paguroida d'autre part, avec celles des Lithodidae et des Paguridae. La famille des Parapaguridae que nous pensons utile de rétablir sur de nouvelles bases morphologiques, exposées ci-dessous, se situe dans la seconde superfamille, celle des Paguroida.

\section{MATERIEL}

Ce travail a été réalisé grâce à un matériel très abondant, provenant de plusieurs grandes expéditions océanographiques : celles du "Siboga" en Indonésie (1899-1900), de 1"“Albatross" aux Hawaï, aux Philippines, au Japon et dans le Pacifique oriental (1889-1910), de la "Galathea" autour du monde (1951-1952). Diverses collections, moins importartes, nous ont également fourni un matériel appréciable : ce sont celles de Th. Mortensen dans l'Indopacifique en 1914, 1922 et 1929 , de K. Sakai au Japon en 1963 et 1964 , de A. Crosnier sur la côte occidentale d'Afrique de 1963 à 1969, de la Fisheries Research Station de Hong Kong de 1963 à 1965, du Wellington Museum (Nouvelle-Zélande) et enfin du Western Australian Museum. En dehors du matériel conservé au Muséum d'Histoire naturelle de Paris, de nombreux spécimens, et notamment les types de la plupart des espèces décrites, appartenant à différents musées, ont pu être examinés, soit sur place, soit par communication.

L'étude et la confrontation de ce matériel nous ont permis d'établir un genre et seize espèces ou sous-espèces nouveaux, et, pour le genre Parapagurus, d'élargir considérablement la distribution géographique de nombreuses espèces, de rectifier un certain nombre d'erreurs d'identification et de mettre plusieurs formes en synonymies.

Le présent travail représente une étude préliminaire, dans laquelle nous mentionnons toutes les espèces décrites 1 ) de la famille des Parapaguridae, avec les références essentielles qui s'y rapportent, la distribution, et certaines remarques indispensables. Nous y décrivons succinctement les formes que nous considérons comme nouvelles, en donnant les illustrations nécessaires à leur identification. La liste détaillée du matériel paraitra dans les volumes consacrés aux Pagurides des expéditions du "Siboga" et de la "Galathea", et dans un travail plus complet sur la famille des Parapaguridae, comportant la redescription et la figuration de toutes les formes connues.

Malgré l'abondance du matériel dont nous avons disposé, certains problèmes de systématique concernant le genre Parapagurus n'ont pu être résolus d'une manière satisfaisante. La plupart des espèces présentent en effet une variabilité considérable, portant sur la longueur relative des appendices céphaliques, la diniension des cornées, la forme et l'ornementation de la main du grand chélipède, la longueur relative des pattes ambulatoires, et enfin la pilosité. En raison de cette variabilité, et de l'existence possible de populations présentant des caractères particuliers liés à leur localisation géographique ou bathymétrique, nous avons dans plusieurs cas hésité à séparer spécifiquement certaines formes : nous avons alors provisoirement considéré qu'il s'agissait de sousespèces, en attendant de disposer d'un matériel

1) Holmes, en 1900, a rattaché au genre Parapagurus une espèce californienne qu'il identifiait à Pagurus mertensii Brandt. Makarov (1938) a montré, d'une part que l'identification de Holmes était erronée, d'autre part que $P$. mertensii Brandt était synonyme de P. hirsutiusculus (Dana).

Le "Parapagurus mertensii" de Holmes, qui reste à nommer, et dont nous avons examiné un exemplaire, n'est pas un Parapagurus et appartient à un genre encore non décrit de la famille des Paguridae. 
plus abondant, susceptible de faire l'objet d'études biométriques.

Les dimensions données dans les descriptions correspondent à la longueur de la carapace, mesurée du sommet de la saillie rostrale jusqu'au bord postérieur des branchiostèges.

Famille des Parapaguridae, Smith, 1879, emend.

\section{Diagnose. -}

Onze paires de branchies, à savoir, de chaque côté, 10 arthrobranchies de $\mathrm{pmx} 3$ à $\mathrm{p} 4$, et une pleurobranchie sur $\mathrm{p} 4$. Parfois une pleurobranchie rudimentaire sur $\mathrm{p} 5$. Lamelles branchiales divisées en deux lobes, ou entières, avec tous les intermédiaires entre ces deux formes.

Ecusson céphalothoracique non rétréci dans sa partie postérieure. Epistome orné de deux épines médianes impaires, la première, toujours présente, située immédiatement en avant du labre (épine labrale), la seconde, qui manque parfois, entre les insertions des pédoncules antennulaires (épine interantennulaire).

Tergites abdominaux entiers, les régions calcifiées non disposées en plaques paires sur chaque segment.

Telson entier, sans constriction transversale médiane.

Premier maxillipède à basipodite élargi et frangé de soies sur son bord externe; exopodite non flagellé.

Coxae des troisièmes maxillipèdes séparées par un large sternite armé d'une paire de tubercules épineux ; région distale de l'ischion de ces appendices nettement plus large que la base du mérus; crista dentata bien développée, régulière; pas de dent accessoire.

Chélipèdes très inégaux (sauf chez Probeebei mirabilis), le droit plus long et plus fort. Pattes ambulatoires p2 et p3 habituellement longues et grêles, à dactyles inermes. P4 subchéliformes, p5 chéliformes.

Chez le mâle, appareil génital pair, orifices sexuels sur les coxae des dernières pattes thoraciques. Spermatophores en ruban, non pédiculés. Pléopodes pairs présent sur les deux premier segments abdominaux (la plupart des Parapagurus, Tylaspis), sur le premier (Probeebei) ou le second seulement (Typhlopagurus), parfois totalement absents. Pl 3 à pl 5 impairs gauches, biramés, à exopodite court.
Chez la femelle, appareil génital impair, par atrophie de l'ovaire droit; en conséquence, un seul orifice sexuel, sur la coxa de la troisième patte thoracique gauche. Pas de pléopodes pairs, pl 2 à pl 4 impairs à gauche, ovifères, à rames croisées; assez souvent un pléopode vestigial à droite sur le deuxième segment abdominal. P1 5 comme chez le mâle chez Parapagurus et Typhlopagurus, ovifère chez Tylaspis et Probeebei.

\section{DISCUSSION. POSITION SYSTEMATIQUE DES PARAPAGURIDAE}

Par plusieurs caractères importants, les Parapaguridae se rapprochent des Paguridae et des Lithodidae, avec lesquels ils doivent être classés dans la superfamille des Paguroida. En effet, l'écartement des coxae des troisièmes maxillipèdes correspond à un élargissement du sternite de ce segment et à une disposition des pièces endophragmales du céphalothorax fondamentalement différente de celle des Coenobitoida 2 ). Le sens de l'hétérochélie est de plus le même chez les Parapaguridae, les Paguridae et les Lithodidae et inverse de celui des Coenobitoida.

L'examen critique des principaux aspects de la morphologie des Parapaguridae montre cependant qu'ils se différencient des autres Paguroida par une série de caractères :

\section{1) Appareil branchial.}

Bien que la formule branchiale des deux familles dans leur ensemble soit identique, la présence d'une pleurobranchie rudimentaire sur p5 chez certains Parapagurus nous paraît importante; elle laisse en effet supposer que dans ce groupe la disparition des pleurobranchies des p2 et p3 a été antérieure à celle des p5. Elle leur est postérieure chez les Paguridae où les formes considérées comme les plus primitives (groupe Pylopaguropsis, voir de Saint Laurent-Dechancé, 1966) possèdent des pleurobranchies sur p2 et p3 mais non sur p5. Rappelons que chez les Coenobitoida on observe 13 ou 14 paires de branchies, dont des pleurobranchies sur p2, p3, p4 et parfois sur p5.

La forme des lamelles branchiales qui avait paru si importante à Smith est, comme l'a bien exprimé Bouvier (cf. ci-dessus, p. 97) dénuée de valeur systématique. Le passage des trichobranchies aux phyllobranchies, d'abord divisées, puis entières, est

2) Nous reviendrons en détail sur cette question dans notre travail définitif. 
commun à l'évolution de tous les Décapodes. La diversification de la forme des lamelles branchiales chez les Parapagurus traduit simplement l'actualité de leur évolution.

\section{2) Carapace}

Chez les Paguridae et les Lithodidae primitifs, l'écusson céphalothoracique est rétréci dans sa partie postérieure et il existe latéralement, entre le sillon cervical et la ligne transversale, un espace losangique, ou "région branchiale postérieure", relativement large. Chez les Parapagurus, la portion dorso-latérale du sillon cervical et la ligne transversale sont presque contiguës, de sorte que les régions branchiales postérieures sont très réduites.

On observe en outre entre Parapaguridae et Paguridae des différences importantes dans la disposition des lignes calcifiées de la région postérieure de la carapace et des branchiostèges.

\section{3) Epistome}

Les épines labrale et interantennulaire des $\mathrm{Pa}$ rapaguridae manquent chez tous les Paguridae et Lithodidae. Dans toute la série des Pagurides, nous ne les avons observées que chez un Pylochelidae, Parapylocheles scorpio Alcock.

\section{4) Abdomen}

Chez les Paguridae, les tergites abdominaux se présentent dans chaque segment sous la forme de plaques latérales paires, faiblement calcifiées; cette disposition se retrouve chez les Lithodidae, où l'abdomen acquiert une calcification secondaire par addition de plaques calcifiées supplémentaires s'ajoutant aux plaques paires primitives. Chez les Parapaguridae, comme d'ailleurs chez les Coenobitoida, les tergites abdominaux sont entiers.

\section{5) Telson}

La constriction transversale médiane qui divise le telson chez la plupart des Paguridae manque toujours chez les Parapaguridae.

\section{6) Premier maxillipède}

L'exopodite des premiers maxillipèdes se termine par un flagelle multiarticulé chez tous les Pagurides, à l'exception des Parapaguridae et Coenobitidae, qui sont des Coenobitoida adaptés à la vie terrestre. Il s'agit probablement d'une convergence. Il convient toutefois de mentionner que le flagelle des pmx1 manque chez les Galathéides du genre Munidopsis, qui vivent comme beaucoup de Parapaguridae à de grandes profondeurs.

\section{7) Troisième maxillipède}

Chez les Paguridae et les Lithodidae, la largeur de l'ischion des troisièmes maxillipèdes diminue progressivement de la base vers l'extrémité distale, dont le diamètre correspond sensiblement à celui de l'article suivant, et la crista dentata s'accompagne presque toujours d'un, parfois de deux, tubercules spiniformes, ou dents accessoires, situés sur la face interne; la disparition de la dent accessoire est toujours corrélative d'une réduction de la crista dentata (genre Iridopagurus, groupe Ostraconotus, cf. de Saint Laurent, 1966 et 1968). Chez les Parapaguridae, l'ischion des pmx3 est élargi sur toute sa longueur, avec l'extrémité distale nettement plus large que le mérus, et la dent accessoire manque, malgré le développement important de la crista dentata. Ce caractère se retrouve chez les Coenobitoida, certains Pylochelidae exceptés.

\section{8) Appareil génital mâle}

La possession de deux paires de pléopodes sexuels fonctionnels est, chez les Paguroida, propre aux Parapaguridae ; des pléopodes pairs rudimentaires persistent chez certains Paguridae primitifs, soit sur le premier, soit sur le deuxième segment abdominal. La régression et la disparition de ces appendices chez certains Parapagurus correspond à une tendance évolutive commune à tous les Pagurides.

\section{9) Appareil génital femelle}

La disparition du tractus génital droit et de l'orifice correspondant, constante chez tous les Parapaguridae, s'observe sporadiquement dans d'autres familles : certaines espèces de Paguristes chez les Diogenidae et, parmi les Paguridae, chez plusieurs Pagurixus et dans le groupe Ostraconotus (cf. de Saint Laurent, 1968).

L'aspect des pléopodes femelles, dont les rames tendent à se croiser, est particulier aux Parapaguridae. Dans cette famille, comme chez les Paguridae, les aufs sont fixés sur le basipodite et sur l'endopodite, l'exopodite restant libre ; ceci oppose les Paguroida aux Coenobitoida, chez lesquels l'exopodite est également ovifère.

Le cinquième pléopode des femelles est identique à celui des mâles chez Typhlopagurus et 
Parapagurus; il est ovifère chez Tylaspis et Probeebei : ceci est la règle chez tous les Pagurides à habitus plus ou moins cancériforme, Birgus (Coenobitidae), Lithodidae, Munidopagurus, Porcellanopagurus, Ostraconotus (Paguridae).

\section{0) Stades larvaires}

Nous ne reviendrons pas ici sur les caractères larvaires des Parapagurus, exposés dans un travail antérieur (de Saint Laurent-Dechancé, 1964), complété par celui de Williamson \& von Levetzow (1967), et dans lequel ont été mises en évidence les profondes divergences entre les zoés des $\mathrm{Pa}$ rapaguridae et celles des Paguridae.

En conclusion, les Parapaguridae nous paraissent constituer, au sein des Paguroida, un groupe distinct et par sa morphologie et par son évolution, détaché assez précocement du rameau ayant donné naissance aux Paguridae et aux Lithodidae, et ayant conservé certains des caractères des Coenobitoida, comme l'absence de dent accessoire sur l'ischion des pmx3 ou la non division par paires des tergites abdominaux. La morphologie des pièces buccales, et en particulier l'absence de flagelle sur l'exopodite des premiers maxillipèdes, leur est par contre particulière.

Groupe homogène et comportant relativement peu d'espèces, les Parapaguridae sont composés du genre Parapagurus, qui compte une quarantaine d'espèces connues, et de trois genres monospécifiques, Tylaspis Henderson, Probeebei Boone et Typhlopagurus gen. nov. Ces trois derniers genres présentent une série d'adaptations à un mode de vie spécialisé, mais leurs caractères fondamentaux (absence de flagelle sur l'exopodite des pmx1, présence d'épines sur l'épistome et de gonopodes chez le mâle, disparition du tractus génital droit chez la femelle) sont ceux des Parapagurus.

Les espèces de cette famille se caractérisent enfin par leur habitat en eau profonde: trois seulement ont été récoltées à moins de 100 mètres de profondeur; la moitié environ des espèces se trouvent dans la zone bathyale à partir de 100 à 500 mètres et jusqu'à plus de 1000 mètres; huit espèces ou sous-espèces ne vivent qu'à partir de 1000 mètres, et au moins quatre d'entre elles sont franchement abyssales. C'est parmi les Parapagurus qu'ont été récoltés les plus profonds de tous les Pagurides, et même de tous les Décapodes connus.
Genre Parapagurus Smith, 1879

Parapagurus Smith, 1879 : 50.

Sympagurus Smith, 1883 : 37.

Espèce-type: Parapagurus pilosimanus Smith, 1879

Diagnose. -

Ecusson céphalothoracique plus ou moins fortement calcifié. Rostre très peu marqué, à sommet arrondi (sauf chez P. sinensis sp. nov.); saillies latérales faibles, spinuleuses ou non.

Pédoncules oculaires de forme très variable, rétrécis vers l'extrémité distale et à cornée réduite chez les espèces de profondeur, parfois très trapus et à cornée dilatée. Ecailles ophtalmiques petites, entières ou denticulées.

Pédoncules antennulaires dépassant toujours l'extrémité des pédoncules oculaires d'au moins la longueur de leur dernier article.

Ecaille antennaire assez longue, spinuleuse sur le bord interne, sauf chez quelques formes de profondeur.

Mandibule à bord incisif entier, avec un denticule médian.

Endopodite de la maxillule avec ou non un lobe externe.

Chélipède droit plus long et plus fort que le gauche, présentant une grande diversité dans la forme ou l'ornementation de la main. Chélipède gauche grêle, carpe et main comprimés latéralement, d'une forme très homogène dans l'ensemble du genre.

Pattes ambulatoires p2 et p3 longues, grêles, habituellement inermes, à l'exception d'une spinule distale sur le bord dorsal du carpe. Dactyles plus ou moins arqués, bordés dorsalement d'une frange de longues soies.

P4 subchéliformes, le propode orné d'une ou de plusieurs rangées de soies squamiformes, suivant les espèces.

Chez le mâle, le plus souvent des pléopodes pairs, plus ou moins développés, parfois rudimentaires, sur les deux premiers segments abdominaux ; ils manquent chez quelques espèces.

Chez la femelle, orifice sexuel unique à gauche. Pas de pléopodes pairs. Pl 2 à $\mathrm{pl} 4$ ovifères, à rames croisées ; souvent un pléopode vestigial à droite sur le deuxième segment abdominal. PI 5 comme chez le mâle.

Telson entier, le bord postérieur plus ou moins fortement asymétrique, sans indentation médiane prononcée, garni de soies spiniformes. 
Lamelles branchiales divisées ou entières, suivant les espèces.

Nous distinguons ici 41 espèces ou sous-espèces, qui peuvent être réparties en trois groupes, principalement caractérisés par la morphologie de la main du grand chélipède.

\section{Groupe pilosimanus}

Ecusson céphalothoracique entièrement calcifié. Main du grand chélipède allongée, à dactyle généralement peu oblique par rapport à l'axe du propode, à bords non cristiformes, et souvent très pileuse.

Pléopodes pairs toujours présents chez le mâle.

Lamelles branchiales le plus souvent divisées en deux lobes, parfois entières. Souvent une pleurobranchie rudimentaire sur $\mathrm{p} 5$.

Ce groupe comprend des formes abyssales, à pédoncules oculaires grêles et à cornées réduites, apparentées à $\boldsymbol{P}$. pilosimanus $\mathrm{Smith}$, et des formes moins profondes, à pédoncules oculaires normaux. Nous y avons inclus arbitrairement Parapagurus spinimanus Balss, 1913, dont nous n'avons pu examiner l'holotype, qui paraît être un spécimen juvénile, et $\boldsymbol{P}$. chuni Balss, forme aberrante adaptée à la vie dans les coquilles de Dentales.

Dix-huit espèces ou sous-espèces :

1. Parapagurus pilosimanus pilosimanus Smith, 1879

(Planche I fig. 1)

Parapagurus pilosimanus Smith, 1879 : 51.

Eupagurus Jacobii A. Milne Edwards, 1880: 42 (pro parte).

Parapagurus pilosimanus, Smith, 1882 : 20, pl. 2 fig. 4 ; 1883 : 33 , pl. 5 figs. $\left.2-2 \mathrm{a}^{3}\right), 4-5$, pl. 6 fig. $1-4 a$; 1884 : 534 (pro parte).

Eupasurus pilimanus, A. Milne Edwards, 1884: 176. Sympagurus Grimaldii A. Milne Edwards \& Bouvier, 1897 : $134 ; 1899$ : 57, pl. 4 figs. $1-5$.

Parapagurus pilosimanus, A. Milne Edwards \& Bouvier, 1899: 57 (pro parte), pl. 4 figs. 1-5; 1900 : 187 (pro parte).

Distribution. - Atlantique, Indopacifique occidental ?, de 500 à 2000 mètres, exceptionnellement jusqu'à 3500 mètres.

Remarques. - Le type de Sympagurus grimaldii est un jeune mâle de $4 \mathrm{~mm}$ de longueur de cara-

8) Les figures 2-2a et 3-3a de la planche 5 ont ét6 interverties (Smith, 1886 : 187). pace dont les lamelles branchiales, incomplètement développées, sont encore entières. Il ne diffère en rien des jeunes pilosimanus typiques, chez lesquels les pattes ambulatoires dépassent l'extrémité du grand chélipède de toute la longueur de leur dactyle.

2. Parapagurus pilosimanus nudus (A. Milne Edwards, 1891)

(Planche I fig. 2)

Eupagurus Jacobii A. Milne Edwards, 1880 : 42 (pro parte).

Parapagurus pilosimanus, Smith, 1884 : 354 (pro parte). Sympagurus nudus A. Milne Edwards, 1891: 131.

Sympagurus nudus, A. Milne Edwards \& Bouvier, 1894 : 67, pl. 10 figs. $18-26$.

Parapagurus pilosimanus, A. Milne Edwards \& Bouvier, 1894: 64 pl. 9 figs. 1-17 (pro parte) ; $1899: 54$ (pro parte); 1900 : 187 (pro parte).

Distribution. - Atlantique, Indopacifique occidental ?, de 2000 à 4000 mètres.

Remarques. - Parapagurus pilosimanus nudus diffère surtout de la forme typique par la main du chélipède droit, plus massive, à dactyle plus incliné sur l'axe du propode, et couverte de tubercules perliformes très réguliers, et par les pattes ambulatoires plus courtes, ne dépassant généralement pas l'extrémité du grand chélipède de plus de la moitié de leur dactyle.

Le type de Sympagurus nudus est un jeune mâle de $6 \mathrm{~mm}$ de longueur de carapace, à pattes ambulatoires dépassant à peine l'extrémité du grand chélipède, et à lamelles branchiales encore pratiquement entières.

\section{Parapagurus pilosimanus scaber Henderson, 1888}

(Planche I fig. 3)

Parapagurus pilosimanus, Smith, 1884 : 354 (pro parte) ; 1886: 643 (pro parte).

Parapagurus abyssorum Henderson, 1888: 87 (pro parte), pl. 9 fig. 2.

Parapagurus abyssorum var. scabra Henderson, 1888 : 89, pl. 9 fig. 3.

Parapagurus pilosimanus var. abyssorum, A. Milne Edwards \& Bouvier, 1899 : 55 (pro parte), pl. 1 fig. 1; 1900 : 191 (pro parte).

nec Parapagurus pilosimanus abyssorum, Faxon, 1895 : 68.

Distribution. - Atlantique, Indopacifique occidental, de 2500 à 5000 mètres. 

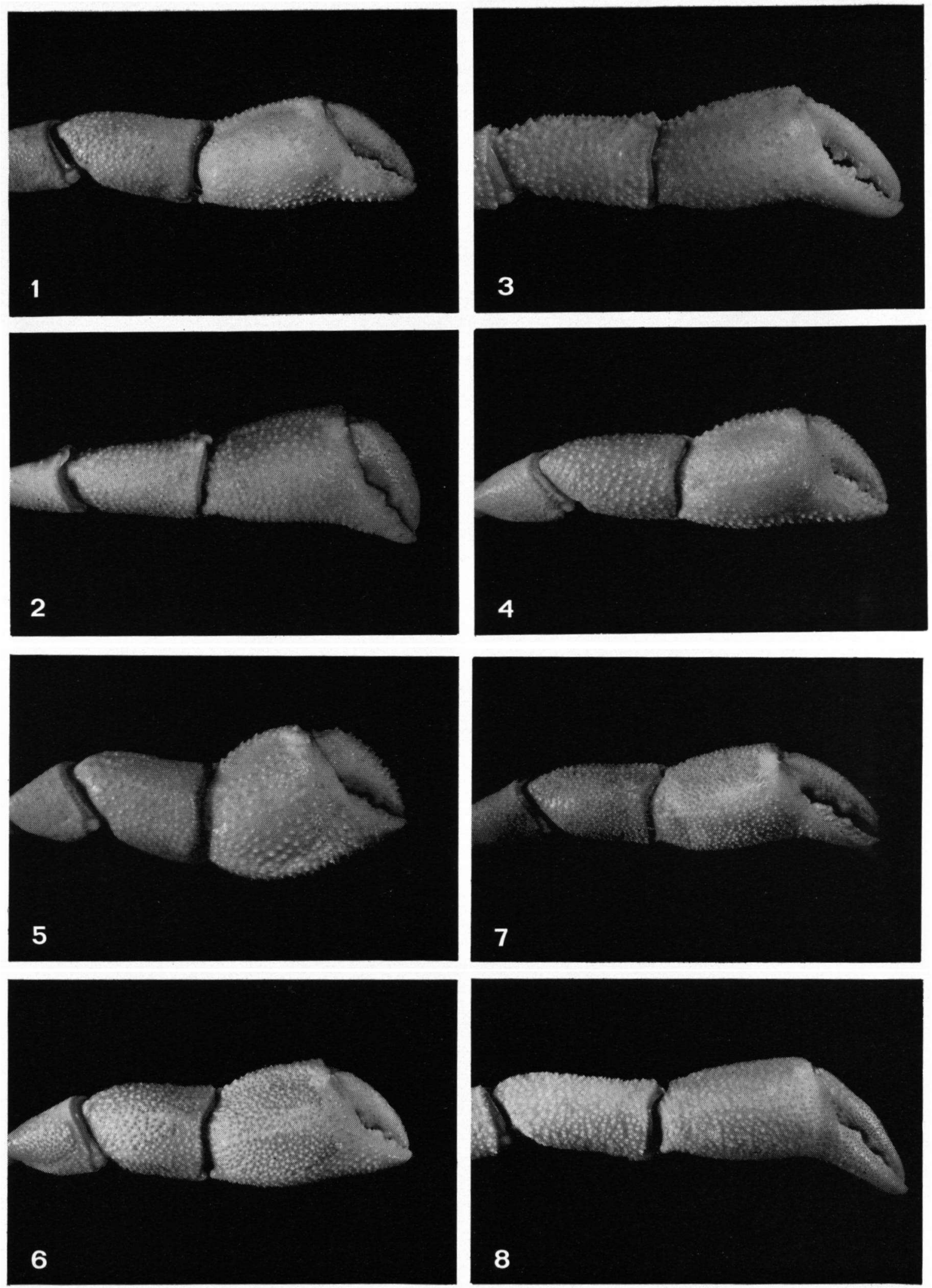

Planche I.

1: Parapagurus pilosimanus pilosimanus Smith.

2: Parapagurus pilosimanus nudus (A. Milne Edwards).

3: Parapagurus pilosimanus scaber Henderson.

5: Parapagurus pilosimanus latimanus Henderson.

6: Parapagurus pilosimanus benedicti ssp. nov.

7: Parapagurus pilosimanus abyssorum Henderson

8: Parapagurus microps sp. nov. 
Remarques. - Confondue dans la description originale de Henderson avec abyssorum, du Pacifique oriental, dont le type provient du large de Valparaiso, cette forme se distingue de pilosimanus et de nudus par les chélipèdes plus grêles, à dactyles plus longs, par la forme de la main droite, infléchie vers l'extérieur, le bord externe du doigt fixe formant avec celui de la paume un angle prononcé. Ce caractère ne s'observe chez pilosimanus et nudus que chez certains mâles de grande taille.

\section{Parapagurus pilosimanus bouvieri Stebbing, 1910}

(Planche I fig. 4)

Parapagurus bouvieri Stebbing 1910 : 357, pl. 18.

Parapagurus pilosimanus, Barnard, 1950: 450 (pro parte).

Distribution. - Afrique du sud (type et "Galathea"), de 350 à 500 mètres.

Remarques. - P. pilosimanus bouvieri, moins profond que la forme typique de l'espèce, ne s'en distingue guère que par les pédoncules oculaires un peu plus longs, cylindriques, et à cornées bien développées.

\section{Parapagurus pilosimanus latimanus Henderson, 1888}

(Planche I fig. 5)

Parapagurus latimanus Henderson, 1888 : 91, pl. 10 fig. 2.

Distribution. - Sud de l'Australie ("Galathea"), Nouvelle-Zélande (Wellington Museum), de 1000 à 2000 mètres.

Remarques. - Cette sous-espèce paraît être la forme australienne et néozélandaise de pilosimanus pilosimanus dont elle diffère surtout par la main droite plus large et plus plate, sur laquelle les tubercules de la face dorsale tendent à se grouper en lignes longitudinales, et par les pattes ambulatoires plus courtes.

Il existe certainement une erreur dans la profondeur de récolte mentionnée par Henderson pour le type (10 fathoms).
6. Parapagurus pilosimanus benedicti ssp. nov. (Planche I fig. 6)

Holotype : \& $16 \mathrm{~mm}$, "Albatross" station 5699, au large de la Californie, $1200 \mathrm{~m}$.

Description. -

Très voisin du pilosimanus pilosimanus atlantique par la forme et la dimension de la main droite, $P$. pilosimanus benedicti s'en distingue par la carapace plus allongée, à région cardiaque très étroite, par les écailles ophtalmiques divisées à leur extrémité et par les pattes ambulatoires plus courtes.

Distribution. - Côte pacifique nord-américaine, de l'Alaska au golfe de Panama (“Albatross"), de 750 à 1600 mètres.

Remarques. - La bidentation de l'extrémité des écailles oculaires n'est pas constante, nous avons observé des écailles entières chez 12 des 68 spécimens examinés, soit environ $17 \%$.

Cette sous-espèce représente la forme pacifique ouest-américaine de pilosimanus pilosimanus dont elle paraît avoir la répartition bathymétrique.

Certains échantillons s'accompagnaient d'une étiquette manuscrite portant la mention "Parapagurus armatus Benedict". Ce nom ne figurant dans aucune des publications de cet auteur, nous avons préféré ne pas le conserver afin d'éviter toute confusion avec Pagurus armatus (Dana), de la même région.

7. Parapagurus pilosimanus abyssorum Henderson, 1888

(Planche I fig. 7)

Parapagurus abyssorum Henderson, 1888: 87 (pro parte).

Parapagurus pilosimanus abyssorum, Faxon, 1895: 68. nec Parapagurus abyssorum Henderson, 1888, pl. 9 fig.

2 ( $=$ P. pilosimanus scaber).

nec Parapagurus pilosimanus var. abyssorum, A. Milne Edwards \& Bouvier, 1899: 55; 1900 : 191 (= P. pilosimanus scaber et $P$. pilosimanus nudus).

Distribution. - Pacifique oriental, de la Californie à Valparaiso, Galapagos ("Albatross", "Galathea"), de 1300 à 2600 mètres.

Remarques. - Le matériel mentionné par Henderson (1888: 87) sous le nom de Parapagurus abyssorum comprend, d'une part des spécimens 
atlantiques ou de l'Indopacifique occidental, identifiables à $P$. pilosimanus scaber, d'autre part ceux de la station $300 \mathrm{du}$ "Challenger", au large de Valparaiso, d'où provient l'exemplaire type utilisé par cet auteur pour sa description. Il semble par contre que l'individu figuré planche 9 fig. 2 soit un $P$. pilosimanus scaber.

$P$. pilosimanus abyssorum prèsente, par rapport aux autres sous-espèces de pilosimanus, une carapace plus fortement calcifiée, notamment dans la région cardiaque et sur la partie antérieure des régions branchiales; les écailles antennaires dépassent très largement l'extrémité des pédoncules antennaires. La main droite ressemble à celle de scaber, mais elle est un peu plus large, et, sur le bord externe, l'angle du doigt fixe avec la paume est moins prononcé. Son ornementation est constituée par des granulations plus denses et beaucoup plus fines.

Nous reconnaissons donc, pour l'instant, sept sous-espèces de Parapagurus pilosimanus Smith, dont la distinction est assez délicate, mais qui correspondent peut-être en fait à des espèces différentes.

Dans l'Atlantique, d'où nous avons examiné des centaines de spécimens, la présence de trois formes distinctes nous paraît indéniable, en dépit des variations individuelles, souvent très importantes, qui se traduisent par le chevauchement interspécifique de certains caractères et qui sont en partie liées, d'une part à la présence chez les mâles de variants sexuels à chélipède droit très allongé et à main infléchie vers l'extérieur, d'autre part, probablement, aux conditions bathymétriques.

Dans l'Indopacifique, si l'on excepte scaber, dont des exemplaires typiques ont été capturés à de grandes profondeurs en différentes localités, latimanus, d'Australie et de Nouvelle-Zélande, relativement bien caractérisé par la forme et l'ornementation de la main droite, et les deux formes américaines benedicti et abyssorum, définies par de bons caractères, l'identification des spécimens est plus difficile. Nous avons provisoirement attribué, soit à pilosimanus, soit à nudus, des exemplaires récoltés au Japon et en Indonésie par 1"'Albatross" et le "Siboga", mais qui appartiennent peut-être à des formes différentes, encore non décrites. Le matériel peu abondant dont nous avons disposé ne nous a pas permis de résoudre cette question.

\section{Parapagurus microps sp. nov.}

(Figures 1, 13 et planche I fig. 8)

Holotype : \& $14 \mathrm{~mm}$, "Albatross" station 4742, Galapagos, $4250 \mathrm{~m}$.

\section{Description. -}

Ecusson céphalothoracique plus long que large, lisse, très faiblement bombé. Saillie rostrale très faible, largement arrondie.

Pédoncules oculaires très écartés, grêles, cylindriques, atteignant tout juste la base du dernier article des pédoncules antennulaires et le milieu du dernier article des pédoncules antennaires. Ecailles ophtalmiques très petites, entières.

Ecailles antennaires droites, inermes, dépassant très légèrement l'extrémité du dernier article des pédoncules.

Chélipède droit (fig. 13) relativement court et grêle. Main allongée, la région digitale infléchie vers l'extérieur.

Les deux chélipèdes et les pattes ambulatoires couverts sur tous leurs articles d'une fine granulation épineuse.

Pléopodes pairs présents, bien développés, chez le mâle.

Lamelles branchiales profondément divisées en deux lobes inégaux.

Distribution. - Trois exemplaires de cette espèce ont été capturés par l'“Albatross" aux îles Gala-

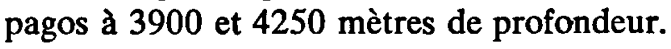

Remarques. - La morphologie de la région antérieure de la carapace et des appendices céphaliques permet de considérer cette forme comme une espèce distincte de $P$. pilosimanus.

Proche des sous-espèces scaber et abyssorum de celle-ci, elle se caractérise par la région rostrale moins saillante, par l'écartement et la gracilité des pédoncules oculaires, par les proportions des appendices céphaliques, et par l'ornementation des appendices.

\section{Parapagurus pictus (Smith, 1883)}

Eupagurus pilimanus A. Milne Edwards, 1880 : 43 (pro parte).

Sympagurus pictus Smith, 1883 : 37, pl. 5 figs. 3$\left.3 a^{4}\right)$, pl. 6 figs. $5-8$.

Distribution. - Côte atlantique nord-américaine, Barbades, de 300 à 560 mètres.

1) et non 2-2a : cf. ci-dessus, p. 102 
10. Parapagurus pilimanus (A. Milne Edwards, 1880)

Eupagurus pilimanus A. Milne Edwards, 1880: 43 (pro parte).

Sympagurus pilimanus, A. Milne Edwards \& Bouvier, $1893: 63$, pl. 5 figs. $8-20$.

nec Parapagurus pilimanus, A. Milne Edwards, 1884 : 176 (= Parapagurus pilosimanus pilosimanus).

Distribution. - Atlantique occidental : Antilles, Floride, de 300 à 400 mètres.

\section{Parapagurus trispinosus Balss, 1911}

Parapagurus arcuatus var. trispinosa Balss, 1911: 3 ; 1912 : 100, fig. 8, pl. 7 fig. 2, pl. 10 fig. 4.

Distribution. - Indopacifique occidental : Afrique du sud (Musée du Cap), Zanzibar, Indonésie ("Albatross", "Galathea"), de 620 à 1400 mètres.

Remarques. - Décrit à l'origine comme une variété de $P$. arcuatus A. Milne Edwards, $P$. trispinosus en est en réalité très éloigné. Proche de $P$. dofleini Balss et de P. brevipes sp.nov., il s'en distingue principalement par les pédoncules oculaires plus grêles et par les écailles ophtalmiques à sommet divisé en deux ou trois spinules.

\section{Parapagurus andersoni Henderson, 1896}

Parapagurus andersoni Henderson, 1896: 529.

Parapagurus andersoni, Ill. Zool. Investigator, pl. 32 fig. 2.

Parapagurus andersoni, Alcock, 1901 : 220.

Parapagurus andersoni var. brevimana Alcock, 1901 : 221.

nec Parapagurus brevimanus Balss, 1911 : 4 ; 1912 : 100, fig. 9 (= P. dimorphus).

Distribution. - Océan Indien : Zanzibar, Somalies, Maldives, détroit de Malacca ("Galathea"), de 700 à 1300 mètres.

\section{Parapagurus dofleini Balss, 1912}

Parapagurus Dofleini Balss, 1912 : 96, fig. 4b ; 1913 : 50, pl. 1 fig. 5 , pl. 2 fig. 3.

Parapagurus ijimai Terao, 1913 : 383, fig. 4.

Sympagurus burkenroadi Thompson, 1943 : 419, fig. 1.

Distribution. - Indopacifique occidental : Zanzibar, Bornéo ("Siboga"), Japon ("Albatross"), Hawaï ("Albatross"), de 350 à 900 mètres.
Remarques. - La comparaison du type de $P$. dofleini, d'une part avec la description et la figure de Terao de $P$. ijimai, d'autre part avec le type de $S$. burkenroadi, nous a permis d'établir l'identité de ces trois espèces.

\section{Parapagurus affinis Henderson, 1888}

Parapagurus affinis Henderson, 1888 : 91, pl. 9 fig. 4.

Distribution. - Indonésie ("Siboga"), Philippines, Hawaï ("Albatross"), de 360 à 900 mètres.

Remarques. - Cette espèce n'était jusqu’à présent connue que par l'holotype femelle du "Challenger". Douze spécimens supplémentaires ont été récoltés par le "Siboga" et l"'Albatross" en Indonésie, aux Philippines et aux Hawaï.

\section{Parapagurus brevipes sp. nov.}

(Figures 2 et 14)

Parapagurus arcuatus var. monstrosus, Balss, 1912 : 99, pl. 10 fig. 3.

Holotype: $q 27 \mathrm{~mm}$, "Siboga" station 12, Bornéo, $289 \mathrm{~m}$.

\section{Description. -}

Ecusson céphalothoracique (fig. 2) sensiblement aussi large que long. Saillie rostrale triangulaire, dépassant le niveau des saillies latérales qui sont à peine indiquées.

Pédoncules oculaires dilatés dans leur moitié distale, atteignant l'extrémité du deuxième article des pédoncules antennulaires et du dernier article des pédoncules antennaires. Ecailles ophtalmiques petites, acuminées, entières.

Ecailles antennaires spinuleuses sur toute la longueur du bord interne, dépassant légèrement le milieu du dernier article des pédoncules.

Chélipède droit (fig. 14) relativement court, trapu. Main ovalaire, face supérieure lisse, les bords de la paume et des doigts ornés de tubercules spiniformes.

Pattes ambulatoires dépassant l'extrémité du grand chélipède d'un peu moins de la moitié de la longueur de leur dactyle.

Pléopodes pairs présents sur les deux premiers segments abdominaux chez le mâle, les pl 1 de dimension réduite.

Lamelles branchiales larges, présentant à leur extrémité un court lobe externe. Une pleurobranchie rudimentaire sur $\mathrm{p} 5$. 

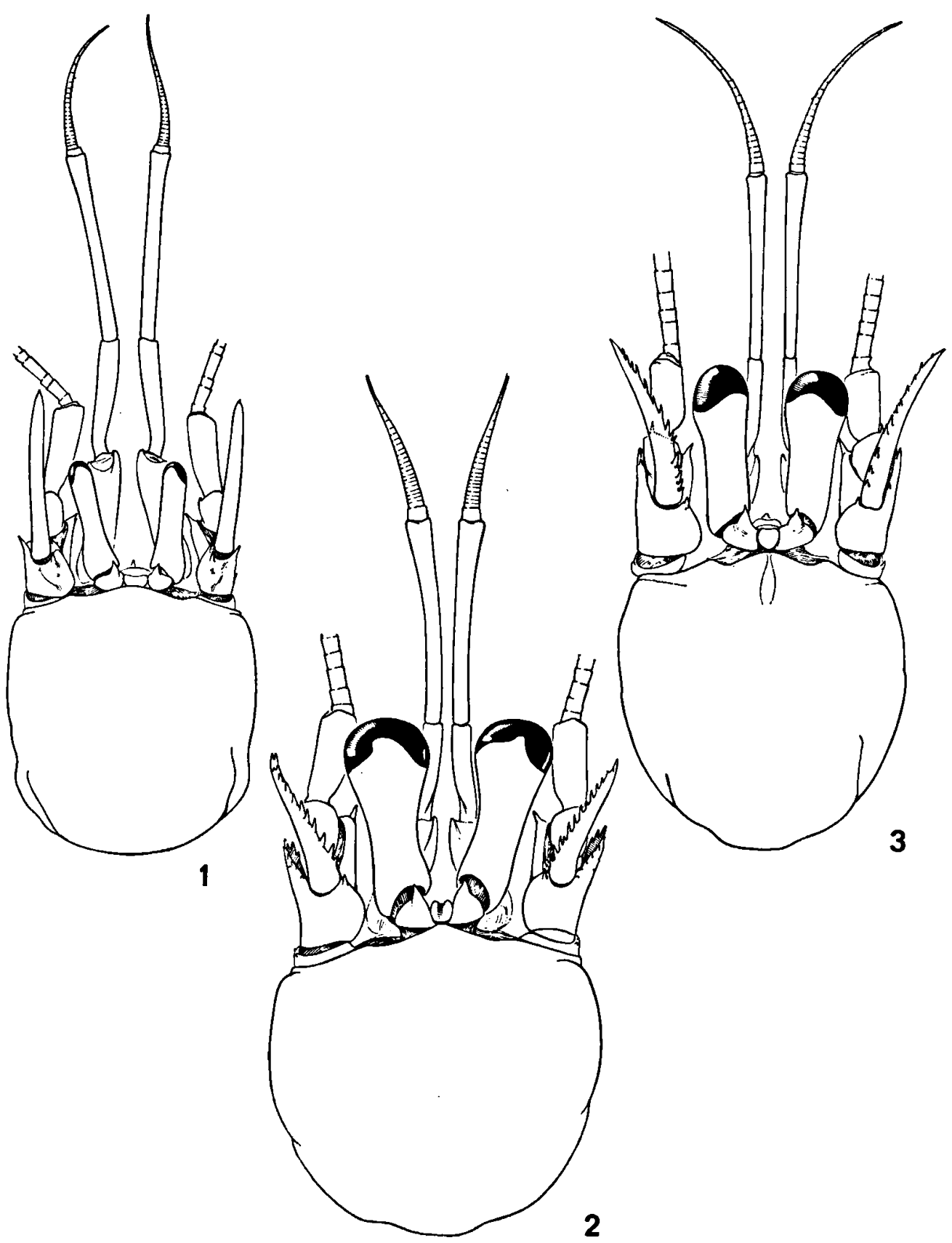

Figures 1-3. - Région antérieure de la carapace et appendices céphaliques: 1, Parapagurus microps sp. nov.,

$\times 6 ; 2, P$. brevipes sp. nov., $X 4 ; 3, P$. africanus sp. nov., $\times 7,2$.

Distribution. - Indopacifique occidental : Zanzibar ("Valdivia"), Indonésie ("Siboga", "Galathea", coll. Mortensen), Philippines ("Albatross"), de 200 à 600 mètres.

Remarques. - Parapagurus brevipes est la plus grande des espèces de Parapagurus actuellement connues, la carapace pouvant atteindre une longueur de $46 \mathrm{~mm}$ chez le mâle et de $36 \mathrm{~mm}$ chez la femelle. C'est aussi la moins profonde des espèces du groupe I, et la plus fréquemment récoltée en Indonésie.

Elle se distingue de $P$. affinis, $P$. andersoni et $P$. trispinosus bar les écailles oculaires entières. de $P$. dofleini par les pédoncules oculaires plus longs, dilatés dans la région cornéenne, et par les appendices thoraciques plus trapus. 
16. Parapagurus spinimanus Balss, 1911

Parapagurus spinimanus Balss, $1911: 1 ; 1912$ : 100, fig. 10 et 23, pl. 9 fig. 2.

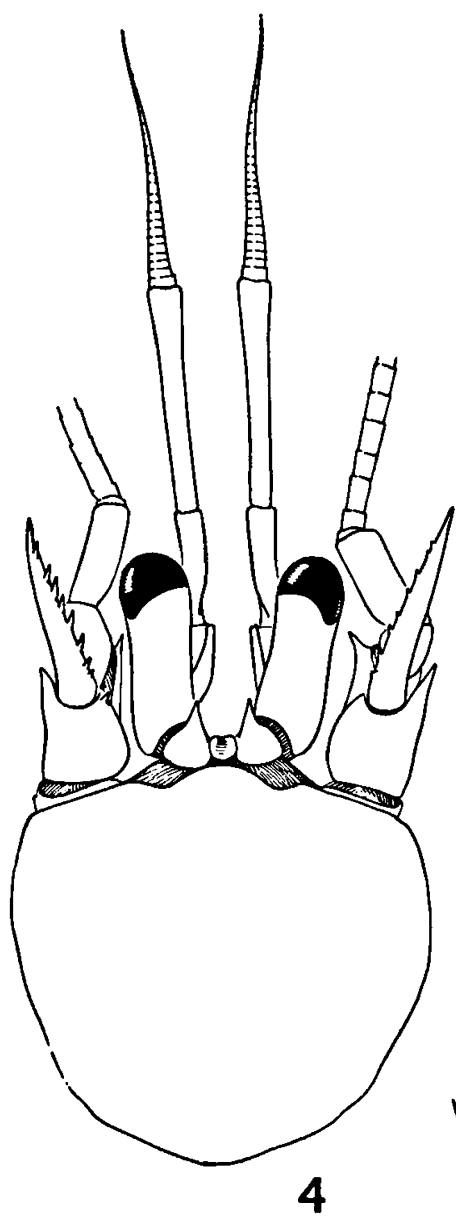

Figures 4-6. - Région antérieure de la carapace et appendices céphaliques: 4, Parapagurus planimanus sp.

sp. nov., $\times 7$.

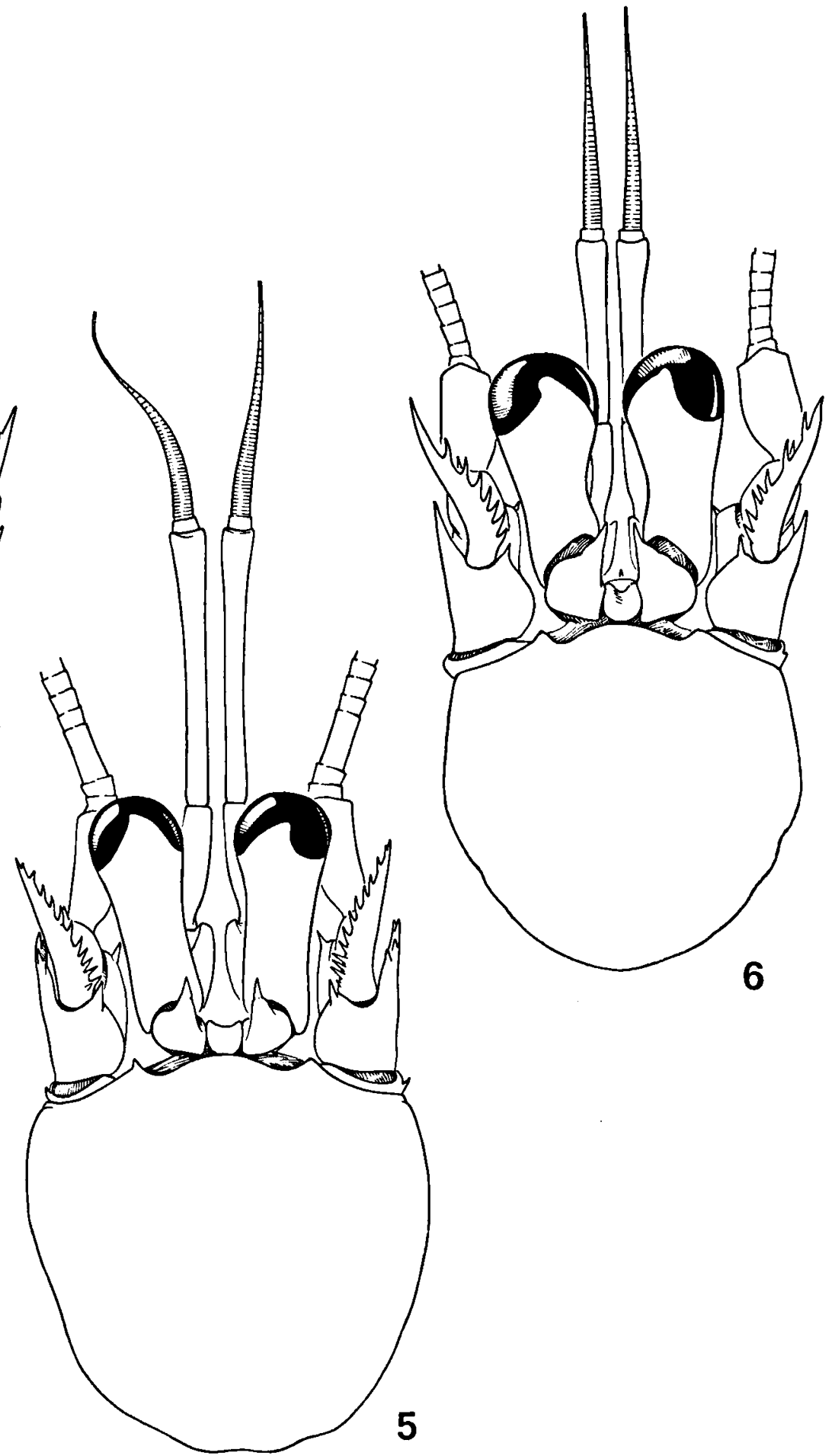

Distribution. - Connu par un seul exemplaire, récolté par la "Valdivia" sur la côte est-africaine, à 277 mètres de profondeur. 
17. Parapagurus chuni Balss, 1911

Parapagurus Chuni Balss, 1911: 3; 1912 : 101, figs. $11-13$ et 23, pl. 9 fig. 2.

Distribution. - Côte est-africaine, à 638 et 977 mètres de profondeur.

\section{Groupe dimorphus}

Ecusson céphalothoracique généralement peu calcifié. Main du chélipède droit plus ou moins grossièrement rectangulaire, à dactyle très oblique par rapport à l'axe du propode, à bords cristiformes, souvent infléchie vers l'intérieur et amincie dans la région externe.

Pléopodes pairs présents ou non chez le mâle.

Lamelles branchiales parfois divisées en deux lobes, le plus souvent entières, avec tous les intermédiaires entre ces deux formes.

Espèces de taille petite ou moyenne.

Dix-huit espèces ou sous-espèces :

A. Formes dont les mâles possèdent des pléopodes pairs.

\section{Parapagurus dimorphus (Studer, 1883)}

Eupagurus dimorphus Studer, 1883 : 24, pl. 2, figs. 11 et 12.

Parapagurus dimorphus, Henderson, 1888 : 86, pl. 10 fig. 1.

? Sympagurus arcuatus var. johnstoni Hale, 1941 : 279, fig. 13.

? Sympagurus arcuatus var. mawsoni Hale, 1941 : 280, fig. 14.

Parapagurus brevimanus Balss, 1911 : 4 ; 1912 : 100, fig. 9.

Distribution. - Hémisphère sud : Amérique du sud ("Calypso"), Tristan da Cunha ("Challenger"), Afrique du sud ("Gazelle", "Galathea"), Nouvelle Amsterdam ("Valdivia"), Tasmanie ("Galathea"), Nouvelle-Zélande (Wellington Museum), de 120 à 500 mètres.

19. Parapagurus arcuatus (A. Milne Edwards \& Bouvier, 1893)

Eupagurus bicristatus A. Milne Edwards, 1880: 43 (pro parte).

Sympagurus arcuatus A. Milne Edwards \& Bouvier, 1893 : 67, pl. 5 figs. $21-28$.

Pylopagurus exquisitus Boone, 1927 : 71, fig. 14.

nec Sympagurus arcuatus var. monstrosus, Alcock, 1905 : 101, pl. 10 fig. 3 (= P. monstrosus Alcock)

nec Parapagurus arcuatus var. trispinosa, Balss, 1912 : 100, pl. 7 fig. 2, pl. 10 fig. 4 (=P. trispinosus Balss) nec Parapagurus arcuatus var. monstrosus, Balss, 1912

99, pl. 10 fig. 3 (= P. brevipes sp. nov.)

nec Sympagurus arcuatus var. diogenes, Hale, 1941

279 ( $=P$. diogenes Whitelegge).

nec Sympagurus arcuatus var. johnstoni Hale, 1941

279, fig. 13 (= ? P. dimorphus Studer)

nec Sympagurus arcuatus var. mawsoni Hale, 1941 :

280, fig. 14 (= ? P. dimorphus Studer).

Distribution. - Antilles, 250 à 400 mètres.

\section{Parapagurus monstrosus Alcock, 1894}

“? Parapagurus monstrosus" Alcock, 1894 : 243.

Sympagurus monstrosus, Henderson, 1896 : 553.

Sympagurus arcuatus var. monstrosus, Alcock, 1905 : 104, pl. 10 fig. 5.

? Eupagurus brevimanus Yokoya, 1933 : 90, fig. 34.

Sympagurus bicristatus, Thompson, 1943 : 418 (pro parte).

nec Parapagurus arcuatus var. monstrosus, Balss, 1912 : 99, pl. 10 fig. 3 (= P. brevipes sp. nov.).

Distribution. - Golfe du Bengale (Indian Museum), golfe d'Aden, Maldives ("John Murray"), Indonésie, Philippines ("Albatross" et coll. Mortensen), Japon (coll. K. Sakai), de 200 à 1000 mètres.

Remarques. - Les spécimens décrits par Yokoya (1933) sous le nom d'Eupagurus brevimanus appartiennent certainement au genre Parapagurus; leur identité avec $\boldsymbol{P}$. monstrosus est probable.

\section{Parapagurus minutus Henderson, 1896}

Parapagurus minutus Henderson 1896 : 531.

Parapagurus minutus, Alcock, 1905 : 101, pl, 10 fig. 3. Parapagurus pilosimanus, Thompson, 1943 : 417 (pro parte).

Parapagurus bicristatus, Thompson, 1943 : 418 (pro parte).

nec Parapagurus minutus, Thompson, 1943 : 417 (= P. bicristatus indicus, Alcock).

Distribution. - Maldives ("Investigator", "John Murray"), Bornéo ("Siboga"), mer de Tasmanie ("Galathea"), de 800 à 2300 mètres.

Une forme très proche, sinon identique, non décrite ici, a été récoltée aux îles Galapagos et dans le golfe de Panama par 1"“Albatross" et la "Galathea" à respectivement 1140 et 938 mètres de profondeur.

\section{Parapagurus diogenes (Whitelegge, 1900)}

Sympagurus diogenes, Whitelegge, $1900: 172$, pl. 24 fig. 3. 
Parapagurus diogenes, Terao, 1913 : 382.

Sympagurus arcuatus var. diogenes, Hale, 1941: 279.

Distribution. - Australie, mer de Chine ("Albatross"), Japon ("Albatross"), de 60 à 180 mètres.

\section{Parapagurus pacificus (Edmondson, 1925)}

Sympagurus pacificus Edmondson, 1925 : 28, fig. 5e-g, pl. 2 fig. C.

Distribution. - Ile de Laysan (Hawaï), 366 mètres. L'espèce n'est connue que par l'holotype mâle, en très mauvais état.

\section{Parapagurus africanus sp. nov.}

(Figures 3 et 15)

Parapagurus bicristatus, Forest, 1961 : 231.

Holotype. - \& $11 \mathrm{~mm}$, Angola, $550 \mathrm{~m}$, A. Crosnier coll. 1968.

\section{Description. -}

Ecusson céphalothoracique (fig. 3) sensiblement aussi long que large. Saillies frontale et latérales peu marquées.

Pédoncules oculaires légèrement dilatés dans leur moitié distale, atteignant presque l'extrémité du deuxième article des pédoncules antennulaires et du dernier article des pédoncules antennaires. Ecailles ophtalmiques petites, entières, acuminées.

Ecailles antennaires arquées, spinuleuses sur le bord interne, dépassant nettement l'extrémité du dernier article des pédoncules.

Chélipède droit (fig. 15) long. Main beaucoup plus longue que large, à dactyle très oblique. Face dorsale faiblement granuleuse ; bord externe cristiforme; sur le bord palmaire interne, on note dorsalement une crête très régulière de tubercules spiniformes, et, ventralement, une seconde crête très atténuée; les deux crêtes sont séparées par un intervalle couvert de tubercules plus ou moins épineux. Face ventrale lisse ou très faiblement granuleuse.

Pléopodes pairs présents, moyennement développés, chez le mâle.

Lamelles branchiales entières, rétrécies au sommet.

Distribution. - Atlantique sud-tropical, au large de l'Angola et du Congo (coll. A. Crosnier), aux environs de 500 mètres de profondeur.

Remarques. - Cette forme est apparentée à $P$. bicristatus bicristatus A. Milne Edwards, dont la répartition dans l'Atlantique est plus nordique. Elle en diffère par la taille moyenne plus grande, les pédoncules oculaires et les écailles antennaires plus longs, et par la crête ventrale du bord palmaire interne de la main, à peine indiquée. Chez bicristatus bicristatus, cette seconde crête est en outre séparée de la première par un espace lisse.

\section{Parapagurus planimanus sp. nov.}

(Figs. 4 et 22)

Holotype : of $12 \mathrm{~mm}$, "Siboga" station 45, mer de Flores, $794 \mathrm{~m}$.

\section{Description. -}

Ecusson céphalothoracique (fig. 4) très légèrement plus large que long. Saillie frontale arrondie, dépassant le niveau des saillies latérales qui sont à peine indiquées.

Pédoncules oculaires assez courts, cylindriques, atteignant à peu près le milieu du deuxième article des pédoncules antennulaires et du dernier article des pédoncules antennaires. Ecailles ophtalmiques petites, acuminées, entières.

Ecailles antennaires presque droites, spinuleuses sur le bord interne, dépassant largement l'extrémité des pédoncules oculaires et atteignant sensiblement celle du dernier article des pédoncules antennaires.

Chélipède droit (fig. 22) massif. Main large, légèrement infléchie vers l'intérieur, à dactyle très oblique, incurvée vers la face ventrale, et comprimée dorso-ventralement. Face dorsale lisse ou faiblement granuleuse, sauf sur les bords, qui sont denticulés. Face inférieure présentant sur la paume une carène oblique, granuleuse, s'étendant de l'angle proximal externe à l'angle distal interne et délimitant une zone externe lisse et très amincie, et une zone interne granuleuse et relativement épaisse. Face inféro-latérale du dactyle excavée.

Pléopodes pairs présents chez le mâle, peu développés.

Lamelles branchiales profondément divisées en deux lobes inégaux.

Distribution. - Indonésie ("Siboga", "Albatross"), de 570 à 800 mètres, mer de Chine méridionale (F.R.S. Hong Kong), de 100 à 600 mètres.

Remarques. - Parapagurus planimanus diffère de la plupart des autres espèces du groupe II par les pédoncules oculaires relativement courts, à cornée non dilatée, et par l'aspect de la face ven- 


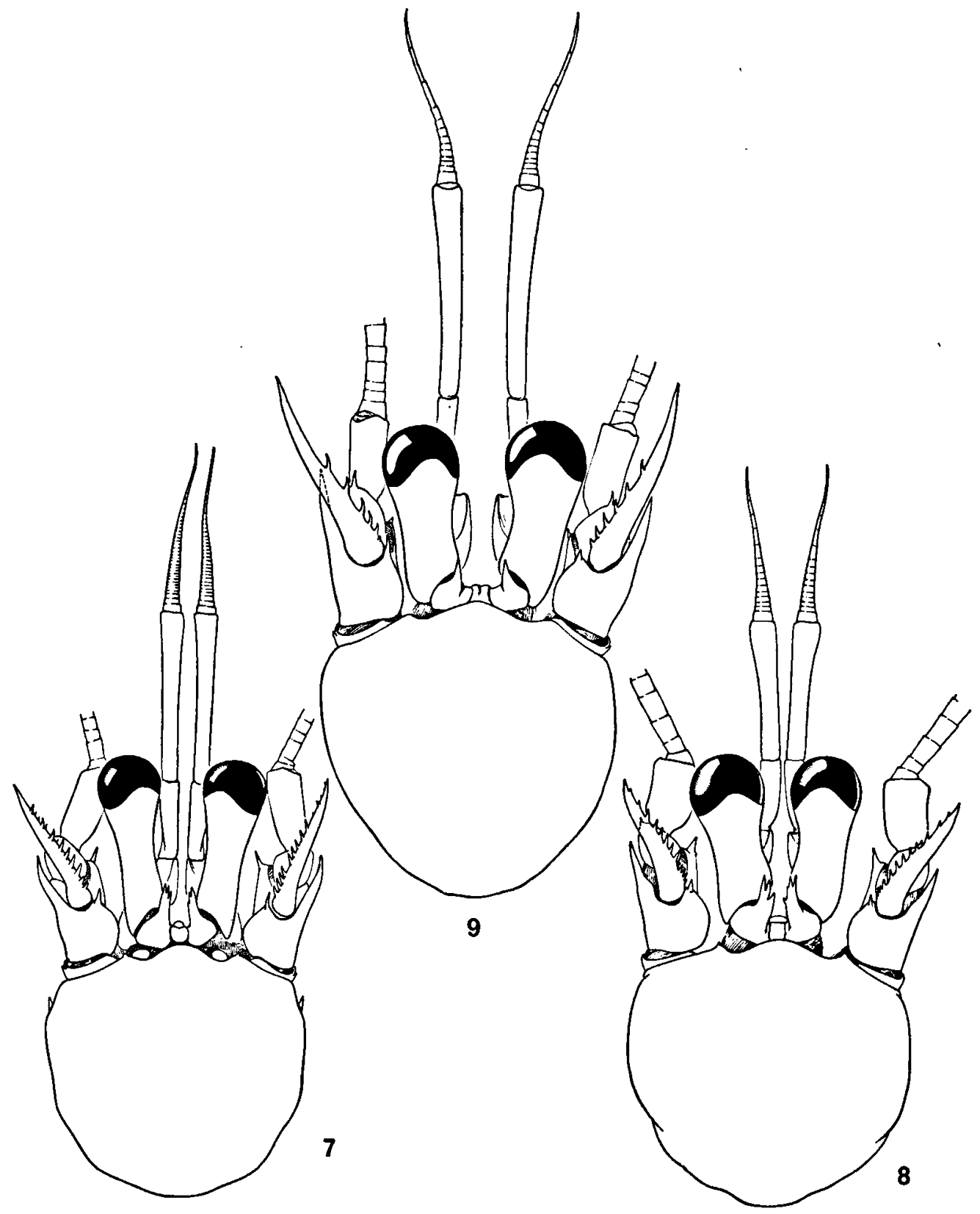

Figures 7-9. - Région antérieure de la carapace et appendices céphaliques: 7, Parapagurus acutus acutus sp.

trale de la main droite, dont la carène oblique est très caractéristique.

Nous rattachons provisoirement à cette espèce plusieurs spécimens récoltés au large de Hong Kong, à plus faible profondeur, chez lesquels la cornée est légèrement dilatée, et la crête ventrale de la main droite très atténuée. et ssp. nov., $\times 4 ; 8, P$. orientalis sp. nov., $\times 6 ; 9$, $P$. haigae sp. nov., $\times 6$.

\section{Parapagurus boletifer sp. nov.}

(Figures 5 et 20)

Holotype : o $14 \mathrm{~mm}$, Tosa Bay (Japon), $250-300 \mathrm{~m}$, K. Sakai coll. 1963.

\section{Description. -}

Ecusson céphalothoracique (fig. 5) un peu plus 


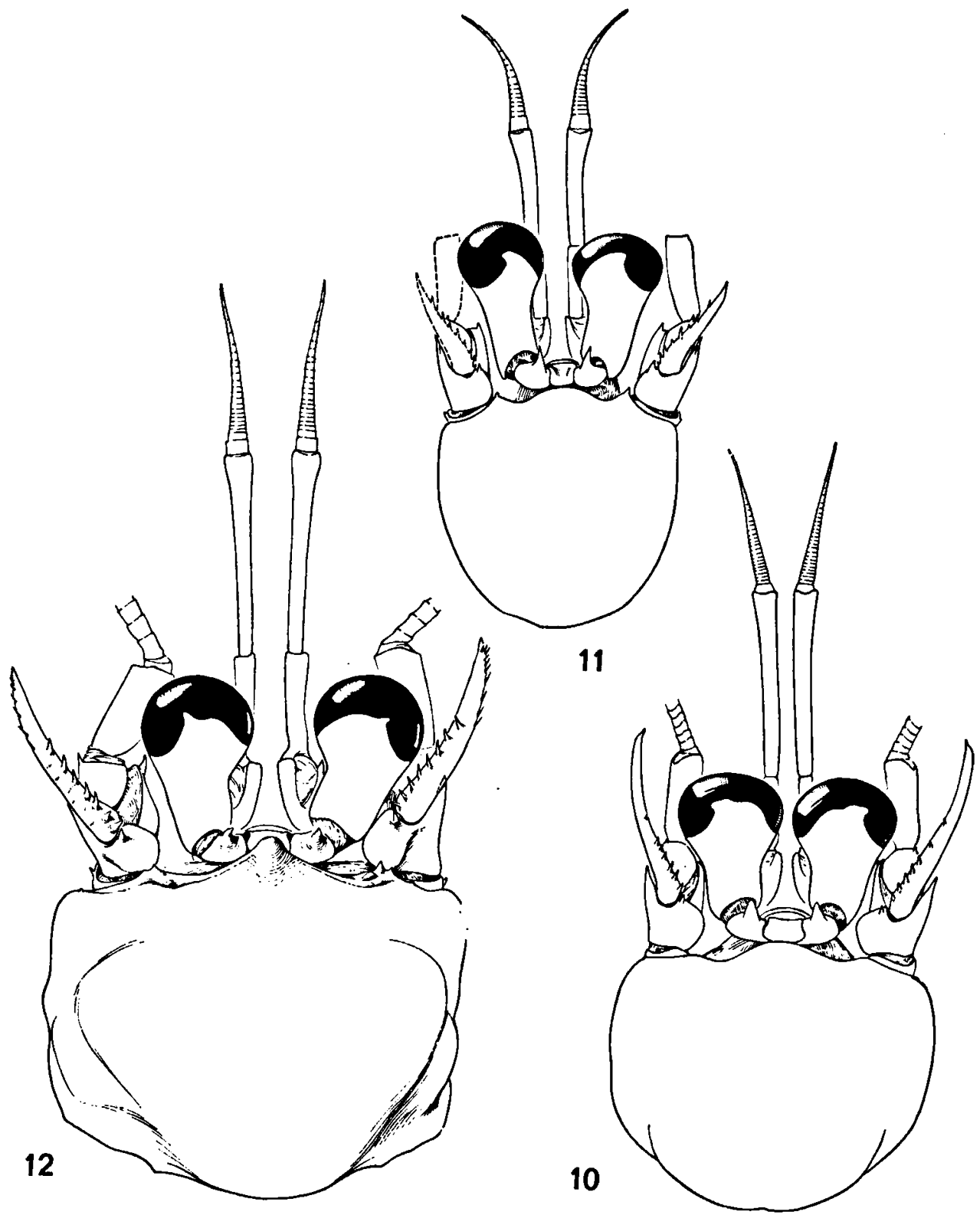

Figures 10-12. - Région antérieure de la carapace et appendices céphaliques: 10, Parapagurus sibogae sp. nov.,

long que large. Saillie frontale largement arrondie, alignée avec les saillies latérales qui sont acuminées.

Pédoncules oculaires longs, légèrement dilatés au niveau des cornées, dépassant de peu ou atteignant tout juste l'extrémité du deuxième article des pédoncules antennulaires et du dernier article des pédoncules antennaires. Ecailles ophtalmiques assez longues, droites, aiguës.

Ecailles antennaires presque droites, dépassant $\times 4,8 ; 11, P$. tuberculosus sp. nov., $\times 9 ; 12, P$. sinensis sp. nov., $\times 6$.

de peu le bord postérieur des cornées.

Chélipède droit (fig. 20) massif. Main très large, fortement infléchie vers l'intérieur, le dactyle formant un angle presque droit avec l'axe du propode. Face supérieure couverte de tubercules épineux serrés, plus forts et plus longs sur les bords. Face ventrale de la paume et des doigts ornée de tubercules arrondis, d'aspect plus ou moins bolétiforme.

Pléopodes pairs bien développés chez le mâle. 
Lamelles branchiales entières, larges, légèrement rétrécies vers leur extrémité apicale.

Distribution. - Japon, 85-300 mètres (coll. K. Sakai et "Albatross").

Remarques. - Apparentée à $\boldsymbol{P}$. dimorphus et à $P$. diogenes, cette espèce s'en distingue immédiatement par l'ornementation de la main droite, dont les tubercules ventraux sont très caractéristiques.

27. Parapagurus rugosus sp. nov.

(Figures 6 et 21)

Holotype : \& $15 \mathrm{~mm}$, "Albatross" station 3859, Hawaï, $253-256 \mathrm{~m}$.

Description. -

Ecusson céphalothoracique (fig. 6) aussi long que large. Saillie rostrale largement arrondie, légèrement dépassant les saillies latérales qui portent une faible spinule apicale.

Pédoncules oculaires assez longs, dilatés dans leur moitié distale, dépassant largement l'extrémité du deuxième article des pédoncules antennulaires et atteignant au plus celle du dernier article des pédoncules antennaires. Ecailles ophtalmiques longues, entières, acuminées.

Ecailles antennaires arquées, garnies de longues épines sur toute la longueur de leur bord interne.

Chélipède droit (fig. 21) relativement fort et trapu. Main épaisse, plus longue que large, à dactyle oblique. Face dorsale couverte de tubercules épineux serrés, plus forts sur la région externe de la paume et sur les doigts. Face ventrale également couverte de granules serrés, franchement épineuse sur le dactyle.

Pléopodes pairs bien développés chez le mâle.

Lamelles branchiales larges, entières.

Distribution. - Archipel des Hawaï ("Albatross"), 240-270 mètres. Trois spécimens seulement sont connus.

Remarques. - Proche de $P$.diogenes et de $P$. boletifer par la forme et les proportions de la carapace et des appendices céphaliques, $P$. rugosus s'en distingue essentiellement par la forme et l'ornementation de la main du chélipède droit.

28. Parapagurus bicristatus gracilis Henderson, 1888

Parapagurus gracilis Henderson, 1888 : 92, pl. 20 fig. 2.
Distribution. - Atlantique occidental, au large de Recife, 640 mètres. Seuls, deux syntypes sont connus.

Remarques. $-P$. bicristatus gracilis diffère de la forme typique par les pédoncules oculaires à cornées plus dilatées, et par la présence de pléopodes pairs chez le mâle.

\section{Parapagurus bicristatus indicus (Alcock, 1905)}

Sympagurus bicristatus var. indicus Alcock, 1905 : 105, pl. 10 fig. 4.

Parapagurus bicristatus, Balss, 1912 : 99, figs. 6-7.

Parapagurus bicristatus, Thompson, 1943: 418 (pro parte).

Parapagurus minutus, Thompson, 1943 : 417.

Distribution. - Maldives, Zanzibar, mer des Indes, Indonésie ("Albatross" "Siboga", "Galathea"), archipel des Hawaï ("Albatross"), de 380 à 1000 mètres.

Remarques. - Nous rattachons, au moins provisoirement, à la variété d'Alcock de nombreux spécimens indonésiens et des Hawaï présentant tous, comme elle, des pléopodes pairs bien développés chez le mâle.

B. Formes dont les mâles sont dépourvus de pléopodes pairs.

30. Parapagurus bicristatus bicristatus (A. Milne Edwards, 1880)

Eupagurus bicristatus A. Milne Edwards, 1880: 43 (pro parte).

Eupagurus ? bicristatus, A. Milne Edwards \& Bouvier, 1893 : 154, pl. 10 figs. $11-12$.

Sympagurus bicristatus, A. Milne Edwards \& Bouvier, 1894: 94, pl. 11 figs. $1-15$.

nec Parapagurus bicristatus, Forest, 1961 : 231 (=P. africanus sp. nov.)

Distribution. - Atlantique occidental : Antilles, et oriental : Açores, Portugal, Sénégal. De 100 à 1300 mètres.

31. Parapagurus ruticheles (A. Milne Edwards, 1891)

Eupagurus ruticheles A. Milne Edwards, 1891 : 206. Sympagurus ruticheles, A. Milne Edwards \& Bouvier, 1894 : 70, pl. 10 figs. $1-14$. 
Distribution. - Atlantique occidental : Açores, Maroc, Sénégal. Archipel des Hawaï ("Albatross"). De 200 à 450 mètres.

Remarques. - D'assez nombreux spécimens de cette espèce, ne différant en rien de ceux de l'Atlantique, ont été récoltés par 1"'Albatross" dans l'archipel des Hawaï.

32. Parapagurus acutus acutus sp. et ssp. nov. (Figures 7 et 18)

Holotype : of $11 \mathrm{~mm}$, "Albatross" station 5222, Philippines, $356 \mathrm{~m}$.

\section{Description. -}

Ecusson céphalothoracique (fig. 7) très légèrement plus large que long. Saillie frontale assez prononcée, dépassant de peu le niveau des saillies latérales, qui sont acuminées. Latéralement, insérées à la limite antérieure des branchiostèges, une paire de petites épines aiguës.

Pédoncules oculaires assez longs, grêles, rétrécis dans leur portion proximale, progressivement dilatés vers les cornées, dépassant de peu l'extrémité du deuxième article des pédoncules antennulaires et atteignant sensiblement celle du dernier article des pédoncules antennaires. Ecailles ophtalmiques longues, leur extrémité divisées en deux, ou plus souvent trois, spinules aiguës.

Ecailles antennaires à bord interne presque droit, fortement denticulé, n'atteignant pas tout à fait l'extrémité des pédoncules.

Chélipède droit (fig. 18) massif. Main plus ou moins infléchie vers l'intérieur, à dactyle très oblique, très amincie du côté externe. Face dorsale faiblement granuleuse, les bords cristiformes, denticulés. Sur la face ventrale du dactyle, une crête longitudinale à sommet arrondi, qui, se prolongeant plus ou moins nettement sur la paume, délimite sur celle-ci une face interne, granuleuse.

Pléopodes pairs absents chez le mâle.

Lamelles branchiales entières.

Distribution. - Philippines ("Albatross"), mer de Chine (F.R.S. Hong Kong), Indonésie (coll. Mortensen), Japon (coll. K. Sakai), de 160 à 560 mètres.

\section{Parapagurus acutus bicarinatus ssp. nov.}

Holotype : of $11 \mathrm{~mm}$, "Albatross" station 5289, Philippines, $314 \mathrm{~m}$.
Description. -

Carapace et appendices céphaliques comme chez P. acutus acutus, à l'exception des pédoncules oculaires et des antennules en moyenne plus courts. Main du grand chélipède peu différente, mais la paume est ornée, du côté interne, de deux crêtes de tubercules épineux bien distinctes, analogues à celles qu'on observe chez $P$. bicristatus.

Pléopodes pairs absents chez le mâle.

Lamelles branchiales entières.

Distribution. — Philippines (“Albatross”), de 275 à 1070 mètres.

\section{Parapagurus acutus hirsutus ssp. nov.} (Figure 19)

Holotype. - of $11 \mathrm{~mm}$, Tosa Bay, coll. K. Sakai, novembre 1963.

Description. -

Carapace et appendices céphaliques comme chez acutus acutus. Main du grand chélipède (fig. 19) plus épaisse, le bord externe cristiforme, mais la région externe beaucoup moins amincie. Face dorsale ornée de tubercules épineux irrégulièrement disposés, beaucoup plus longs et plus forts que les granules de la forme typique. Les dents qui garnissent les bords de la paume et des doigts sont moins nombreuses, plus fortes, et irrégulières.

Pléopodes pairs absents chez le mâle.

Lamelles branchiales entières.

Pilosité générale assez forte.

Distribution. - Mer de Chine, Philippines ("Albatross"), Japon (coll. K. Sakai), 240-400 mètres.

Remarques. - Dans les trois formes que nous rattachons à $P$. acutus sp. nov., on note une grande variabilité dans la longueur relative des pédoncules oculaires, dans l'aspect de l'extrémité des écailles oculaires, dans la forme et l'ornementation du grand chélipède. L'impossibilité d'identifier à l'une ou à l'autre les spécimens dépourvus de grand chélipède nous a incitée à les décrire sous un même nom spécifique, et à les considérer, au moins à titre provisoire, comme des sousespèces.

$P$. acutus se distingue de l'ensemble des espèces du groupe II, à l'exception de $\boldsymbol{P}$. orientalis, décrit ci-dessous, par la denticulation des écailles ophtalmiques, la présence des épines latérales de la ca- 

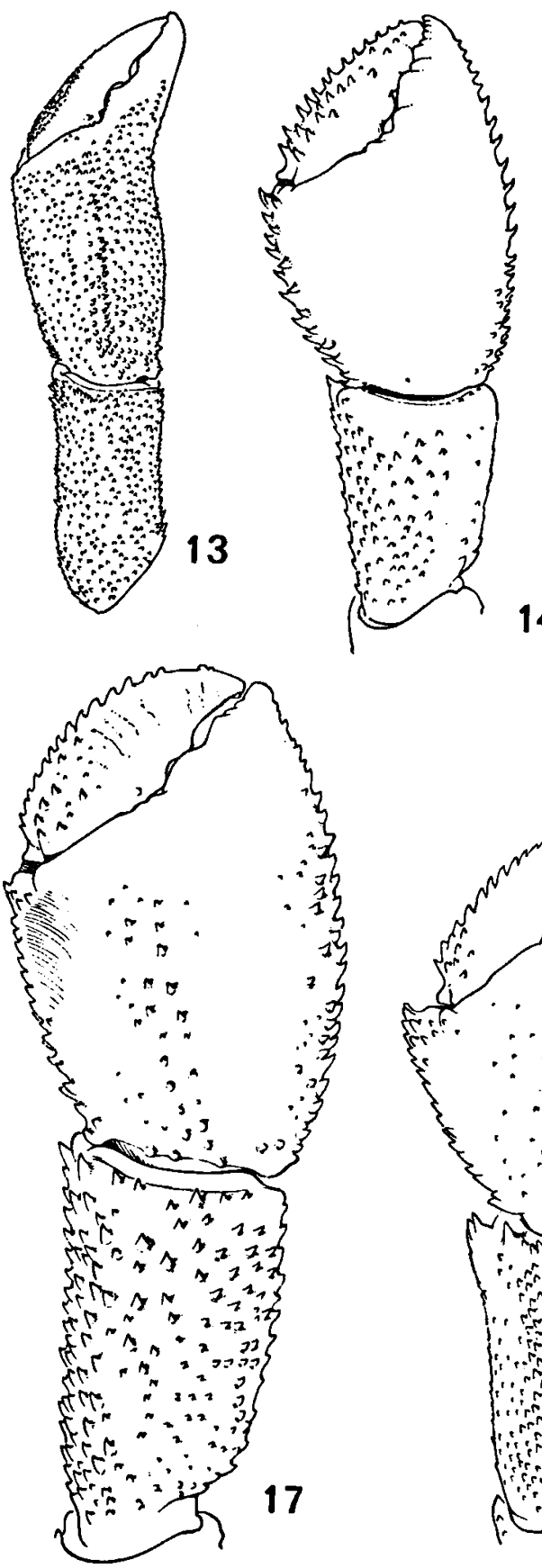

14
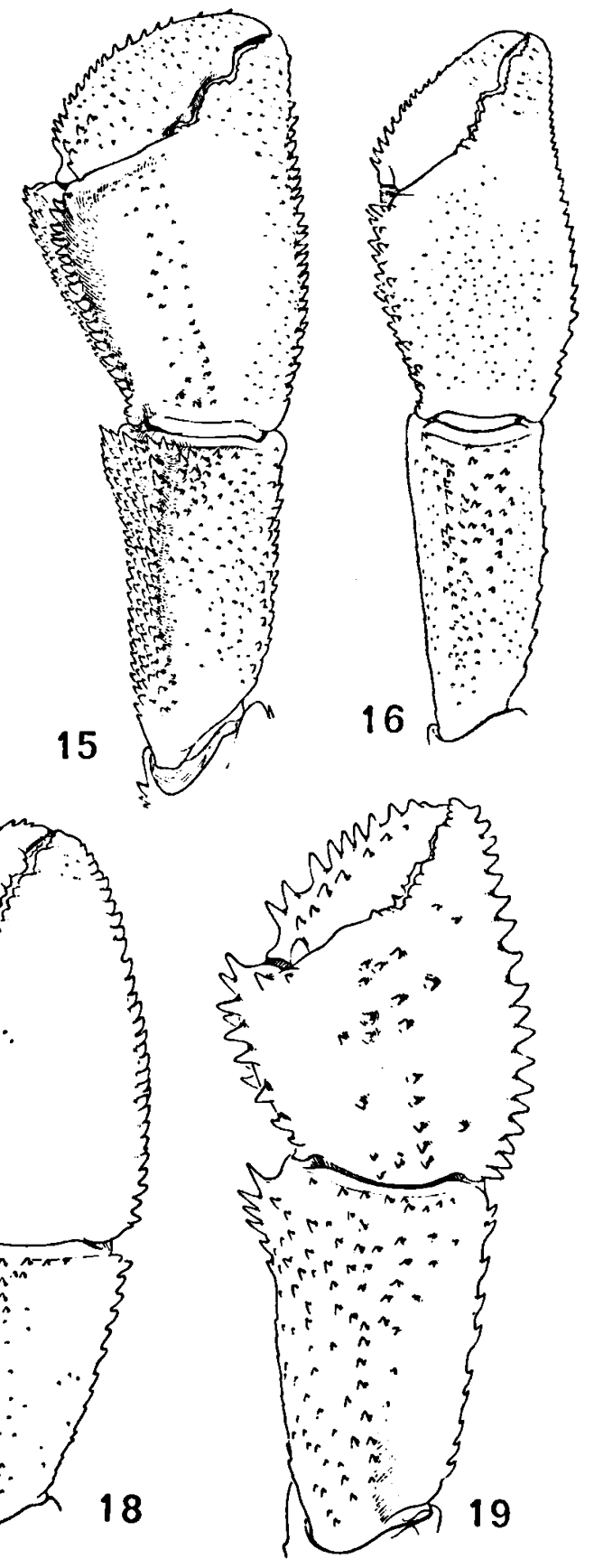

Figures 13-19. - Extrémité du chélipède droit: 13, Parapagurus microps sp. nov., $\times 2,5 ; 14, P$. brevipes sp. nov., $X 2 ; 15, P$. africanus sp. nov., $X 4,5 ; 16, P$. orien-

rapace, et la disparition des pléopodes pairs chez le mâle.

\section{Parapagurus orientalis sp. nov.}

(Figs. 8 et 16)

Holotype : of $5 \mathrm{~mm}$, "Albatross" station 5289, Philippines ou mer de Chine, $314 \mathrm{~m}$. talis sp. nov., $\times 9 ; 17, P$. haigae sp. nov., $\times 7 ; 18$, $P$. acutus acutus sp. et ssp. nov., $X 5 ; 19, P$. acutus hirsutus sp. et ssp. nov., $\times 4,5$.

Description. -

Ecusson céphalothoracique (fig. 8) très légèrement plus large que long. Saillie frontale arrondie, située au niveau des saillies latérales qui sont acuminées.

Pédoncules oculaires amincis vers leur milieu, dépassant de peu l'extrémité du deuxième article 
des pédoncules antennulaires et du dernier article des pédoncules antennaires. Ecailles ophtalmiques longues, se terminant par 3 ou 4 épines aiguës.

Ecailles antennaires n'atteignant pas l'extrémité des pédoncules oculaires, fortement denticulées sur le bord interne.

Chélipède droit (fig. 16) allongé, main environ deux fois plus longue que large chez le mâle type, l'axe du dactyle légèrement oblique. Face dorsale garnie vers le centre de très fins granules, disparaissant sous une pilosité relativement abondante. Bords cristiformes, régulièrement denticulés. Face inférieure de la paume lisse dans la région externe, plus renflée et granuleuse du côté interne.

Pléopodes pairs absents chez le mâle.

Lamelles branchiales entières, rétrécies au sommet.

Remarques. - Les quelques exemplaires que nous rattachons à cette espèce nouvelle sont d'une taille très inférieure à la taille moyenne des trois sous-espèces de $P$. acutus, dont elle paraît par ailleurs très proche. Elle en diffère essentiellement par la forme de la main du grand chélipède, beaucoup plus allongée, chez la femelle comme chez le mâle, et à dactyle moins incliné par rapport à l'axe du propode.

Distribution. - Philippines ("Albatross"), Moluques ("Siboga"), Indonésie ("Galathea" et coll. Mortensen), de 300 à 575 mètres.

\section{Parapagurus haigae sp. nov.}

(Figures 9 et 17)

Holotype : \& $6 \mathrm{~mm}$, Allan Hancock Foundation Station 99-339, golfe de Californie, $225-255 \mathrm{~m}$.

\section{Description. -}

Ecusson céphalothoracique (fig. 9) sensiblement aussi long que large. Saillie frontale dépassant le niveau des saillies latérales.

Pédoncules oculaires dilatés dans leur moitié distale, n'atteignant pas l'extrémité du deuxième article des pédoncules antennulaires, ni celle du dernier article des pédoncules antennaires. Ecailles ophtalmiques relativement longues, entières, très aiguës.

Chélipède droit (fig. 17) massif. Main environ une fois et demie plus longue que large chez le mâle type. Face dorsale avec quelques petits tubercules spiniformes sur la région palmaire interne, au voisinage du bord externe et à la base du dactyle. Face ventrale faiblement granuleuse, amincie dans la région externe; région digitale avec une excavation délimitée par deux carènes à sommet arrondi, sur le doigt fixe et sur le dactyle.

Pléopodes pairs absents chez le mâle.

Lamelles branchiales entières, très étroites.

Remarques. - Nous avons le plaisir de dédier cette espèce nouvelle à $\mathrm{J}$. Haig, qui nous en a communiqué de nombreux spécimens. Comme chez d'assez nombreuses espèces de Parapagurus, la main droite, de forme à peu près constante chez la femelle, s'allonge considerablement chez certains mâles, où elle peut atteindre plus de deux fois sa largeur. $P$. haigae présente des affinités avec $P$. arcuatus A. Milne Edwards, des Antilles, mais elle s'en distingue par les écailles antennaires plus longues, par l'excavation ventrale de la région digitale de la main, et par l'absence de pléopodes pairs chez le mâle.

Distribution. - Golfe de Californie (Allan Hancock Foundation Stations), golfe de Panama ("Galathea"), de 55 à 250 mètres.

\section{Groupe gracilipes}

Ecusson céphalothoracique bien calcifié, large. Pédoncules oculaires courts, fortement dilatés dans la région cornéenne. Main du grand chélipède fusiforme, plus ou moins régulièrement ovalaire, à bords cristiformes et à dactyle peu incliné par rapport à l'axe du propode. Pattes ambulatoires souvent grêles et très longues, à dactyles faiblement arqués.

Pléopodes pairs du mâle toujours présents, plus différenciés que chez les autres espèces du genre qui en possèdent; pl 2 parfois munis d'un court exopodite.

Cinq espèces 5 ) :

37. Parapagurus gracilipes (A. Milne Edwards, 1891)

Sympagurus gracilipes A. Milne Edwards, 1891 : 132. Sympagurus gracilipes, A. Milne Edwards \& Bouvier, 1894 : 68 , pl. 9 figs. $18-34$.

5) Une sixième espèce de ce groupe, proche de $P$. macrocerus Forest, a été récemment découverte au large de l'Angola. 
Distribution. - Atlantique nord-oriental, Açores ("Talisman", "Princesse Alice") de 400 à 1000 mètres, archipel des Hawaï ("Albatross"), de 250 à 730 mètres.

\section{Parapagurus macrocerus Forest, 1955}

Parapagurus macrocerus Forest, 1955 : 101, fig. 22; pl. 3 figs. $1-7$.

Distribution. - Atlantique oriental tropical, de 140 à 280 mètres.

39. Parapagurus sibogae sp. nov.

(Figures 10 et 23)

Holotype : $q 15 \mathrm{~mm}$, "Siboga" station 12, Java, $289 \mathrm{~m}$.

Description. -

Ecusson céphalothoracique (fig. 10) légèrement plus large que long. Saillie frontale largement arrondie, saillies latérales à peine indiquées.

Pédoncules oculaires courts et trapus, à cornées très dilatées, dépassant légèrement l'extrémité du deuxième article des pédoncules antennulaires, mais n'atteignant pas celle du dernier article des pédoncules antennaires. Ecailles ophtalmiques courtes, acuminées, entières.

Ecailles antennaires arquées, spinuleuses sur la moitié proximale de leur bord interne, dépassant légèrement l'extrémité des pédoncules.

Chélipède droit (fig. 23) fort, à main ovalaire, environ une fois et demie plus longue que large chez la femelle type, plus allongée chez le mâle. Face dorsale lisse, à bords épineux, couverte d'une fine pilosité. Face ventrale faiblement granuleuse.

Pléopodes pairs bien développés chez le mâle, pl 2 munis d'un court exopodite.

Lamelles branchiales entières.

Remarques. - Proche de $P$. macrocerus Forest, de l'Atlantique africain, et de $P$. gracilipes A. Milne Edwards, connu de l'Atlantique oriental et des Açores, mais dont nous avons examiné de nombreux spécimens des Hawaĩ, $P$. sibogae s'en distingue principalement par le chélipède droit plus fort, à main plus large et plus régulièrement ovalaire.

Distribution. - Indonésie ("Siboga" et coll. Mortensen) ; mer de Chine (F.R.S. Hong Kong), Japon (coll. K. Sakai), de 200 à 475 mètres de profondeur.
40. Parapagurus sinensis sp. nov.

(Figures 12 et 24)

Holotype : of $14 \mathrm{~mm}, 16^{\circ} 19,3^{\prime} \mathrm{N}, 114^{\circ} 29^{\prime} \mathrm{E}, 200 \mathrm{~m}$ (F.R.S. Hong Kong, Cr. 7, st. 55).

\section{Description. -}

Ecusson céphalothoracique (fig. 12) nettement plus large que long. Rostre relativement saillant, dépassant largement les saillies latérales qui sont bidentées. Région postérieure de la carapace et portions dorsales des branchiostèges calcifiées. Abdomen réduit, logé dans une valve de Mollusque Lamellibranche recouverte d'une Actinie, les deux premiers tergites calcifiés.

Pédoncules oculaires forts, largement dilatés dans leur moitié distale, n'atteignant pas l'extrémité du deuxième article des pédoncules antennulaires, ni celle du dernier article des pédoncules antennaires. Ecailles ophtalmiques petites, entières, dressées.

Ecailles antennaires arquées, spinuleuses sur les deux tiers de leur bord interne, sensiblement aussi longues que les pédoncules.

Chélipède droit (fig. 24) long et grêle. Main, chez le mâle, plus de deux fois plus longue que large. Face dorsale couverte de courtes soies entre lesquelles on distingue deux rangées longitudinales de petites épines peu serrées, les bords épineux et plus ou moins cristiformes. Face ventrale très légèrement granuleuse.

Patte p2 dépassant l'extrémité du chélipède droit de presque la moitié du dactyle; propode comprimé latéralement, dactyle long et très grêle, presque droit. Patte p3 semblable, mais nettement plus courte.

Pléopodes pairs bien développés chez le mâle, pl 1 avec un court exopodite.

Lamelles branchiales entières.

Remarques. - Par l'aspect des appendices céphaliques et des chélipèdes, $P$. sinensis se rapproche quelque peu de $P$. gracilipes A. Milne Edwards et de $P$. macrocerus Forest. Il s'en distingue cependant par de nombreux caractères et notamment par la calcification de la carapace, qui n'est pas recouverte par une coquille, et par la réduction notable de l'abdomen.

L'élargissement de l'écusson céphalothoracique, le développement du rostre, la réduction des écailles oculaires d'une part, de l'abdomen d'autre part, annoncent chez cette espèce une tendance à la carcinisation que nous retrouverons chez $T y$ laspis et, plus accentuée encore, chez Probeebei. 


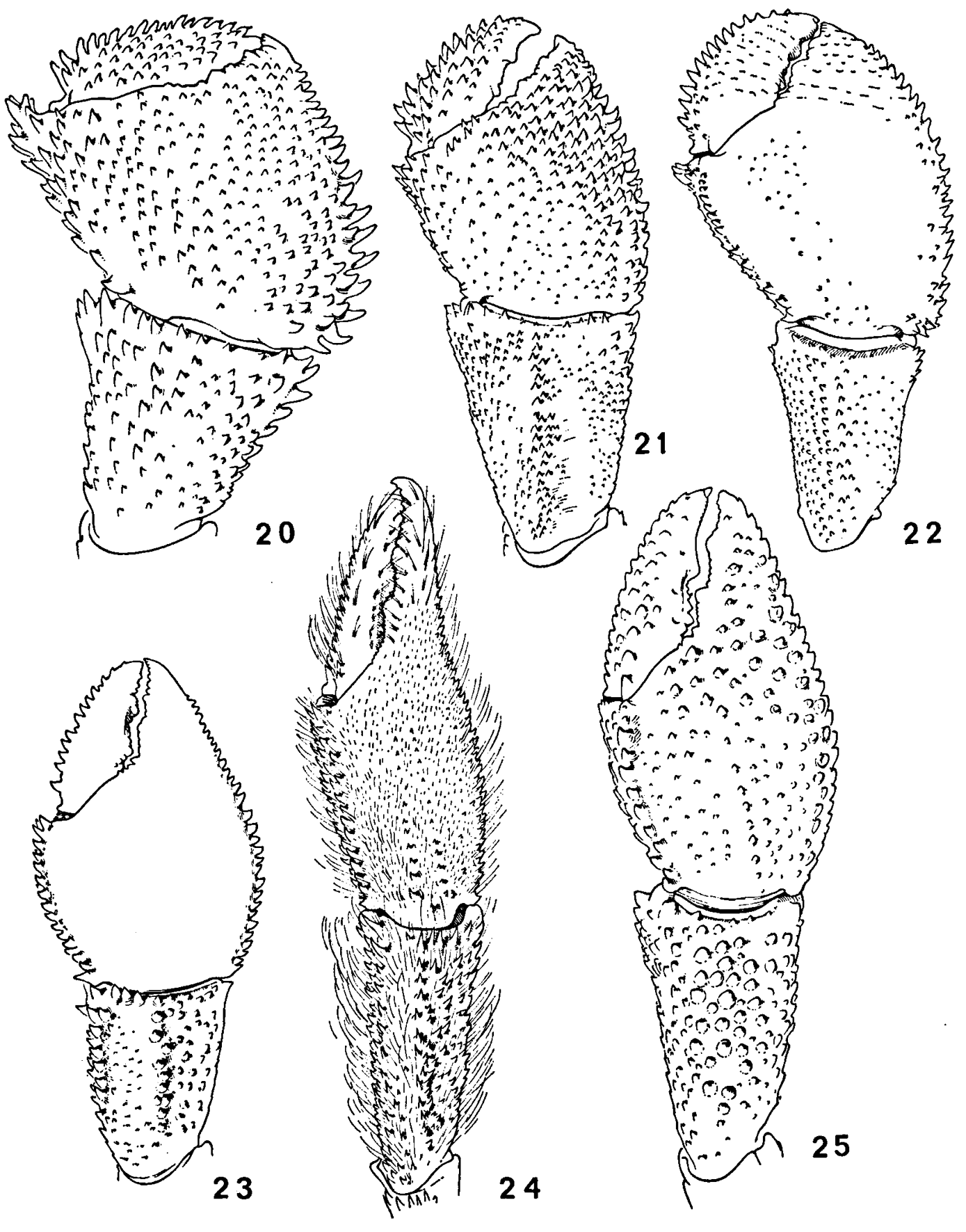

Figures 20-25. - Extrémité du chélipède droit: 20, Parapagurus boletifer sp. nov., 4,5; 21, $P$. rugosus sp. nov., $X 3 ; 22, P$. planimanus sp. nov., $\times 4,5 ; 23, P$. sibogae sp. nov., $\times$ 4,5; 24, $P$. sinensis sp. nov., $\times$ 4,5; $25, P$. tuberculosus sp. nov., $X 7,5$. Les soies n'ont été représentées que sur la figure 24 . 
Distribution. - Mer de Chine méridionale, 200 mètres. Seuls l'holotype mâle et un paratype femelle sont connus.

\section{Parapagurus tuberculosus sp. nov.}

(Figures 11 et 25)

Holotype: o $6 \mathrm{~mm}$, “Albatross" station 3938, île de Laysan (Hawaï), 270-300 m.

\section{Description. -}

Ecusson céphalothoracique (fig. 11) sensiblement aussi long que large. Saillie rostrale faible, ne dépassant pas les saillies latérales qui sont acuminées.

Pédoncules oculaires relativement longs, à cornées dilatées, dépassant l'extrémité du deuxième article des pédoncules antennulaires et du dernier article des pédoncules antennaires. Ecailles ophtalmiques petites, entières, faiblement divergentes.

Ecailles antennaires spinuleuses sur toute la longueur de leur bord interne, atteignant à peu près le milieu du dernier article des pédoncules.

Chélipède droit (fig. 25) long. Main régulièrement ovalaire, presque deux fois plus longue que large. Face supérieure couverte de tubercules spiniformes peu serrés, faibles sur la paume, plus forts sur les bords et au niveau des doigts. Face ventrale régulièrement convexe, très faiblement granuleuse.

Lamelles branchiales assez larges, présentant une encoche distale du côté externe.

Remarques. - La position de cette espèce est douteuse : elle diffère des autres formes du groupe III par les pédoncules oculaires un peu plus longs et moins forts, par les écailles antennaires beaucoup plus courtes, et par l'ornementation plus prononcée de la face dorsale de la main droite, dont les bords sont aussi moins amincis. La découverte du mâle permettrait d'examiner le développement des pléopodes pairs et amènerait peut être à la placer plutôt dans le groupe I.

Distribution. - Iles Hawaï, 220-300 mètres. L'espèce n'est connue que par la femelle type, en très mauvais état, et par un chélipède droit isolé récolté à la station 4101 de l'“Albatross".

Genre Typhlopagurus nov. gen.

Espèce-type: Typhlopagurus foresti $\mathrm{sp}$. nov.

Diagnose. -

Ecusson céphalothoracique calcifié, à bords antérieurs spinuleux. Région postérieure de la carapace, branchiostèges et abdomen membraneux ou très faiblement calcifiés. Bord frontal avec saillie rostrale à peine indiquée et beaucoup plus faible que les saillies latérales.

Bord postérieur du telson avec une encoche médiane large et profonde.

Pédoncules oculaires épineux, cornées absentes. Mandibules à bord interne denticulé.

Un lobe externe sur l'endopodite de la maxillule.

Chélipèdes très inégaux.

P4 subchéliformes, p5 chéliformes.

Pléopodes pairs absents sur le premier segment abdominal chez le mâle, présents sur le second segment.

Une seule espèce :

Typhlopagurus foresti sp. nov.

(Figures 26-29)

Holotype : \& $6 \mathrm{~mm}$, "Albatross" station 5582, Philippines, $1630 \mathrm{~m}$.

Description. -

Ecusson céphalothoracique (fig. 26) plus long que large, saillie rostrale à peine indiquée et beaucoup plus courte que les saillies latérales qui sont très marquées, larges, et à sommet divisé. Sur la partie antérieure de l'écusson, les régions latérales sont ornées de petites spinules, devenant plus fortes sur les bords.

Pédoncules oculaires rapprochés à leur base, brusquement rétrécis vers leur tiers proximal, du côté interne, jusqu'à leur extrémité qui est acuminée; leur bord interne est garni d'une rangée de fortes épines. Ils atteignent presque l'extrémité du deuxième article des pédoncules antennulaires et de l'avant-dernier article des pédoncules antennaires. Ecailles ophtalmiques réduites à de simples plaques calcifiées.

Ecailles antennaires arquées, fortement spinuleuses sur leur bord interne, atteignant presque l'extrémité du dernier article des pédoncules.

Une forte épine interantennulaire (cf. supra, p. 99).

Bord incisif des mandibules denticulé sur toute sa longueur.

Maxillule avec endopodite garni de longues soies sur le bord externe et muni d'un lobe externe, assez long, non recourbé et glabre.

Article principal de l'exopodite des deuxièmes 

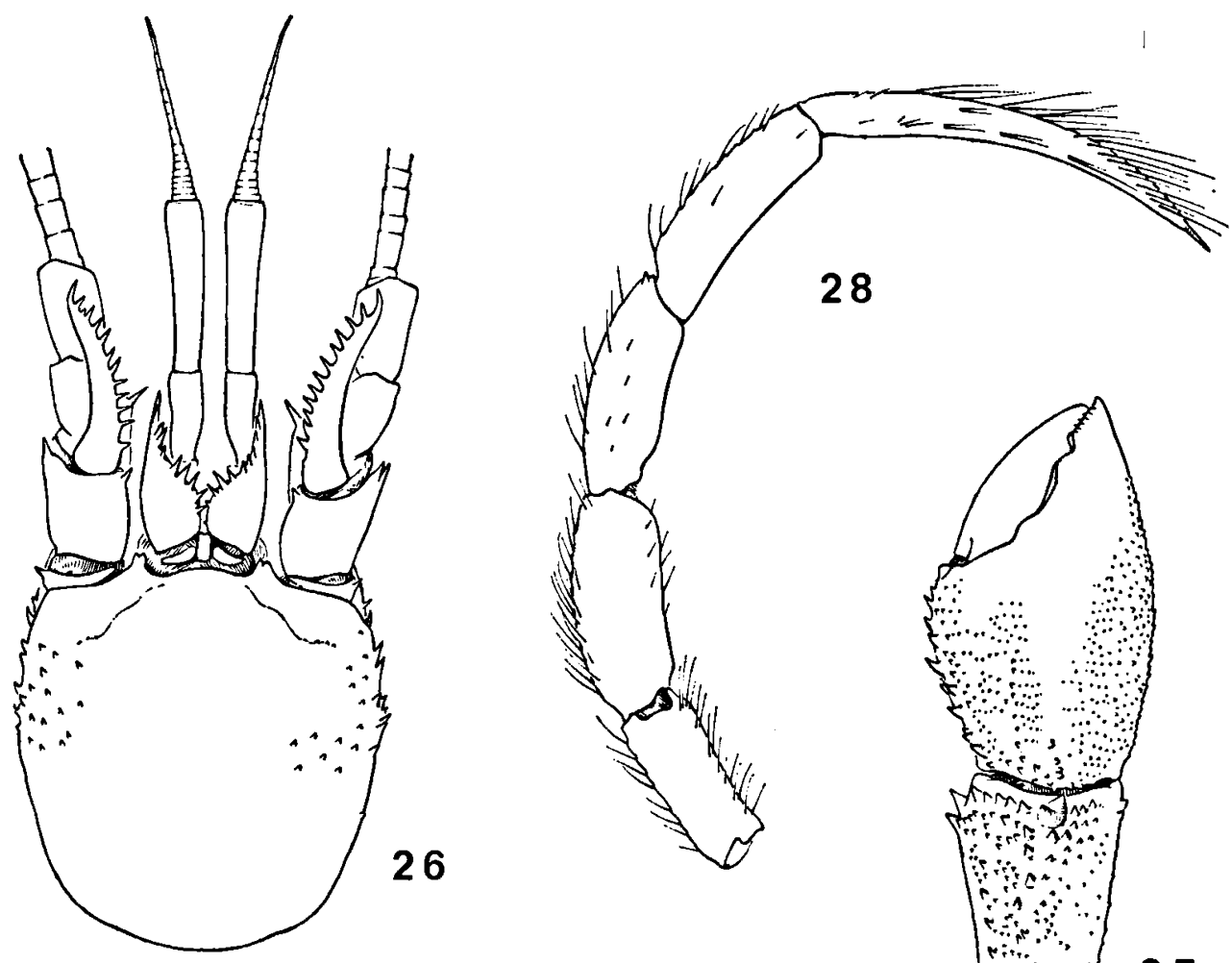

28

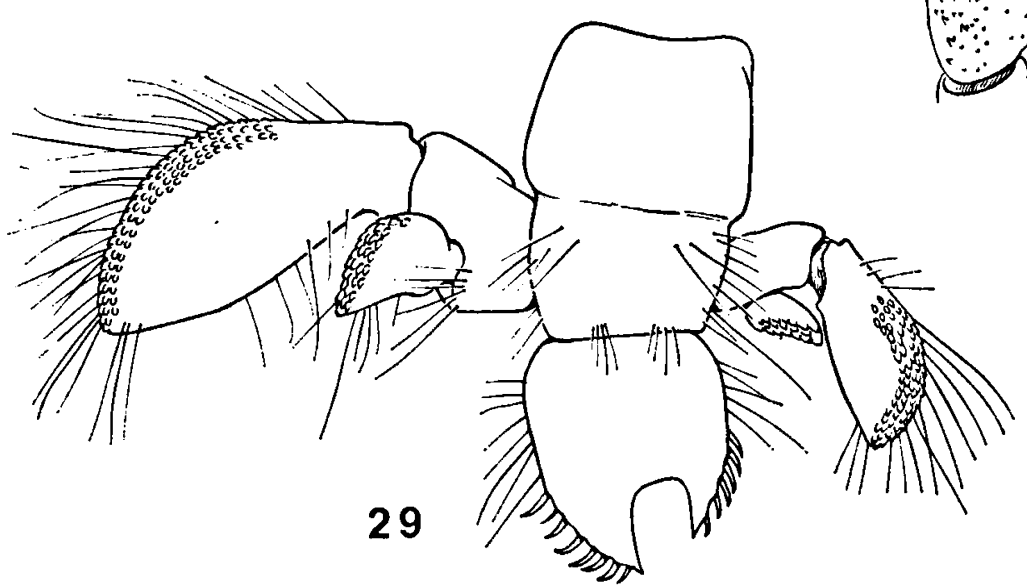

Figures 26-29. - Typhlopagurus foresti gen. nov., sp. nov.: 26 , région antérieure de la carapace et appendices céphaliques, $\times 11 ; 27$, extrémité du chélipède droit, $\times$

maxillipèdes long et grêle, dépassant l'endopodite.

Troisième maxillipède avec exopodite également long et grêle ; sur l'endopodite, une épine distale sur le bord externe du mérus et du carpe.

Chélipèdes très inégaux, le droit beaucoup plus long et plus fort. Main droite (fig. 27) ovalaire, effilée vers l'extrémité des doigts. Face dorsale bombée, la base et les régions latérales de la paume ornées de granules épineux, plus forts dans la région proximale, progressivement atténués vers
7; 28, deuxième patte ambulatoire, $\times 7 ; 29$, telson et uropodes, $\times 16$.

la région digitale où ils disparaissent complètement ; bord interne de la paume spinuleux. Face inférieure lisse.

Chélipède gauche très semblable à celui des Parapagurus. Main comprimée latéralement, à région digitale plus longue que la région palmaire, dont le bord interne est spinuleux.

Pattes ambulatoires p2 (fig. 28) et p3 dépassant de peu l'extrémité du grand chélipède, inermes, à l'exception d'une spinule distale sur le bord supé- 
rieur du carpe. Dactyles relativement courts et trapus, bordés dorsalement d'une frange de longues soies.

Pattes p4 à extrémité subchéliforme, le bord inférieur du propode garni d'une rangée de soies squamiformes.

Pattes p5 chéliformes.

Chez le mâle, pléopodes pairs absents sur le premier segment abdominal. P1 2 pairs bien développés, composés de deux articles. Pléopodes 3 à 5 impairs, biramés, à endopodite court.

Chez la femelle, aucun pléopode pair, pl 2 à pl 4 ovifères, à rames croisées; pl 5 comme chez le mâle.

Telson (fig. 29) présentant une encoche médiane postérieure large et profonde, le divisant en deux lobes inégaux dont les bords externes sont ornés de fortes soies.

Lamelles branchiales profondément divisées.

Remarques. - Le genre Typhlopagurus, établi pour $T$. forest $i$, remarquable espèce nouvelle, que nous sommes heureuse de dédier au Professeur J. Forest, est, sous bien des rapports, proche de Parapagurus, dont il possède, en dehors des pédoncules oculaires épineux et dépourvus de cornées, l'habitus général. Il en diffère cependant par une série de caractères tels qu'on ne peut le considérer simplement comme un Parapagurus à pédoncules oculaires modifiés :

1) l'écusson céphalothoracique est spinuleux sur les bords ;

2) les saillies latérales du bord antérieur de la carapace dépassent de beaucoup le niveau du rostre ;

3) les écailles ophtalmiques sont rudimentaires ;

4) la mandibule est denticulée sur toute la longueur de son bord interne ;

5) le lobe externe de l'endopodite de la maxillule est plus développé que chez les Parapagurus qui en possèdent un ; le bord externe de l'endopodite est frangé de longues soies ;

7) les exopodites des $\mathrm{pmx} 2$ et $\mathrm{pmx} 3$ sont très longs et grêles ;

8) on observe une épine distale sur le carpe de pmx3 ;

9) les pléopodes pairs du mâle manquent sur le premier segment abdominal, mais sont bien développés sur le second ; chez Parapagurus, les deux paires existent ensemble, ou manquent toutes les deux ;

10) le bord postérieur du telson présente une indentation très profonde.
Mais le caractère le plus frappant du nouveau genre concerne les pédoncules oculaires, sur lesquels nous n'avons pu distinguer aucune trace de cornée, et dont l'armature épineuse, très singulière, semble unique chez les Pagurides, si l'on excepte un représentant de la famille des Pylochelidae, Parapylocheles scorpio Alcock ; chez ce dernier toutefois, la spinulation, qui affecte également le bord interne, est plus discrète, et il existe une cornée terminale. Il s'agit là, très probablement, d'un phénomène de convergence.

Typhlopagurus foresti n'est pas comme Probeebei mirabilis Boone et Tylaspis anomala (cf. infra, p. 120), récoltés aux environs de 4000 mètres, une forme particulièrement profonde, puisqu'il a été capturé par l'“Albatross" à un niveau où vivent de nombreuses espèces de Parapagurus à pédoncules oculaires normaux.

Distribution. - $T$. foresti n'est connu que par trois spécimens, deux mâles et une femelle, recueillis en une seule station de l" Albatross", dans les parages des îles Philippines, par 1620 mètres de profondeur.

\section{Genre Tylaspis Henderson, 1885}

Tylaspis Henderson, $1885: 900 ; 1888: 81$. Espèce-type : Tylaspis anomala Henderson, 1885.

\section{Diagnose. -}

Carapace entièrement calcifiée, à l'exception des branchiostèges qui sont membraneux, élargie dans sa partie postérieure. Rostre triangulaire, plus court que les pédoncules oculaires.

Abdomen membraneux, sauf le tergite 2, faiblement calcifié. Telson grossièrement rectangulaire.

Pédoncules oculaires cylindriques, à cornée normalement développée. Ecailles oculaires absentes.

Chélipèdes très inégaux, le droit beaucoup plus fort. P3 considérablement plus longues que les p2. P4 subchéliformes, le propode dépourvu de soies squamiformes sur le bord ventral. P5 chéliformes.

Chez le mâle, pléopodes pairs présents, bien développés sur les deux premiers segments abdominaux, pl 3 à pl 5 biramés, courts.

Chez la femelle, pléopode 2 vestigial à droite, pl 2 à pl 5 gauches ovifères. Uropodes longs et grêles.

Une seule espèce : 
Tylaspis anomala Henderson, 1885

Tylaspis anomala Henderson, 1885 : 900, fig. 329 ; 1888 : 81 , pl. 8 fig. 5.

Distribution. - Le type a été dragué par le "Challenger" dans le Pacifique sud $\left(32^{\circ} 36^{\prime} \mathrm{S}\right.$, $137^{\circ} 43^{\prime} \mathrm{W}^{6}$ ) par 4350 mètres de profondeur. Quatre nouveaux spécimens figurent dans les récoltes de 1"“Albatross", stations 4605 et 4701, aux environs de 4000 mètres.

\section{Genre Probeebei Boone, 1926}

Probeebei Boone, 1926a:73.

Planopagurus Wolff, 1960 : 169.

Espèce-type : Probeebei mirabilis Boone, 1926.

\section{Diagnose. -}

Carapace entièrement calcifiée, épineuse, élargie dans sa partie postérieure. Rostre grêle, allongé, plus long que les pédoncules oculaires. Tergites abdominaux bien individualisés, calcifiés, le second plus long et plus large que les suivants.

b) et non pas $137^{\circ} 43^{\prime} \mathrm{E}$, comme le mentionne Henderson (1888: 194).
Pédoncules oculaires cylindriques, à cornée normale. Ecailles ophthalmiques présentes, réduites à une petite épine.

Chélipèdes subégaux, beaucoup plus courts que les pattes ambulatoires p2 et p3. P3 considérablement plus longues que les p2. P4 à extrémité subchéliforme, le propode dépourvu de soies squamiformes. P5 chéliformes.

Chez le mâle, pléopodes pairs présents, unisegmentés, sur le premier segment abdominal. Pl 2 à pl 5 vestigiaux.

Chez la femelle, pas de pléopodes pairs, pl 2 à pl 5 ovifères.

Uropodes longs et grêles.

Une seule espèce connue :

\section{Probeebei mirabilis Boone, 1926}

Probeebei mirabilis Boone, 1926a : 72 ; 1926b : 73, fig. Planopagurus galathea Wolff, 1960: 169, 177, fig.

Probeebei mirabilis, Wolff, 1961 : 12, figs. 1-10.

Distribution. - Océan Indien (holotype), Pacifique oriental ("Galathea", "Albatross"), 1145 à 4335 mètres de profondeur.

\section{REMERCIEMENTS}

Nous remercions ici les responsables des différents musées qui ont bien voulu nous confier l'étude de leurs collections de Pagurides, ou mettre à notre disposition des types ou des spécimens dont l'examen était nécessaire à la réalisation de ce travail. Une liste des institutions où sont conservés les exemp'aires sur lesquels nous avons fondé notre étude sera donnée dans notre travail définitif. Nous tenons cependant à remercier dès à présent
J. H. Stock, du Zoölogisch Museum d'Amsterdam, PaysBas, T. Wolff, de l'Universitetets Zoologiske Museum de Copenhague, Danemark, et F. A. Chace, de la Smithsonian Institution de Washington, U.S.A., qui, en nous confiant le matériel des expéditions du "Siboga", de la "Galathea" et de l'"Albatross", ont été à l'origine de cette révision.

\section{BIBLIOGRAPHIE}

Alcock, A., 1894. Natural history notes from H.M. Indian Marine Survey Steamer "Investigator", Commander R. F. Hoskyn, R.N., commanding, (2) 1. On the results of deep-sca dredging during the season $1890-91$ (continued). Ann. Mag. nat. Hist., (6) 13 : $225-245$.

-, 1901. A descriptive catalogue on the Indian deep-sea Crustacea Decapoda Macrura and Anomala, in the Indian Museum. Being a revised account of the deep-sea species collected by the Royal Indian Marine Survey Ship Investigator : $1-286+i-i v$, pls. $1-3$. (Calcutta).

-, 1905. Catalogue of the Indian decapod Crustacea in the Indian Museum, 2. Anomura, 1. Pagurides. i$\mathrm{xi}+1-197$, pls. 1-16. (Calcutta).

Alcock, A. \& A. R. S. ANDERSON, 1897. Illustrations of the zoology of the Royal Indian Marine Surveying Steamer "Investigator". Crustacea, 5, pls. 28-32. (Calcutta).
Balss, H., 1911. Neue Paguriden aus den Ausbeuten der deutschen Tiefsee-Expedition "Valdivia" und der japanischen Expedition Prof. Doflein's. Zool. Anz., 38 : 1-9, figs. 1-17.

-, 1912. Paguriden. In : Carl Chun (éd.), Wissenschaftliche Ergebnisse der deutschen Tiefsee-Expedition auf dem Dampfer "Valdivia" 1898-1899, 20 (2) : 85124, figs. $1-26$, pls. $7-11$.

-, 1913. Ostasiatische Decapoden, 1. Die Galatheiden und Paguriden. Abh. bayer. Akad. Wiss. (math.phys. K1.), Suppl. 2 (9) : i-ix + 1-101, figs. 1-54, pls. 1-2.

一, 1957. Decapoda, 8. Systematik. Bronns Klasscn Ordn. Tierreichs, 5 (1. Abteilung, 7. Buch, 12. Lief.): 1505-1672, figs. 1131-1199.

Barnard, K. H., 1950. Descriptive catalogue of South African decapod Crustacea. Ann. S. Afr. Mus., 38 : 1-837, figs. $1-154$.

Boone, L., 1926a. Unusual deep-sea Crustacea - some 
forms secured by the Arcturus Oceanographic Expedition. Bull. N.Y. zool. Soc., 29 (2) : 69-72.

- 1926b. A new family of Crustacea. Preliminary technical description. Bull. N.Y. zool. Soc., 29 (2): 73.

-, 1927. Crustacea from tropical East American seas. Scientific results of the first oceanographic expedition of the "Pawnee" 1925. Bull. Bingham oceanogr. Coll., 1 (2) : $1-147$, figs. $1-33$.

Bouvier E.-L., 1891. Sur les branchies des Paguriens. Ann. Sci. nat. (Zool.), (7) 11 : 400.

EDMondson, C. M., 1925. Crustacea of tropical central Pacific. Bull. Bishop Mus. Honolulu, 27: 1-62, figs. $1-8$, pls. $1-4$.

FAXON, W., 1895. Reports on an exploration off the west coasts of Mexico, Central and South America, and off the Galapagos Islands, in charge of Alexander Agassiz, by the U.S. Fish Commission steamer "Albatross", during 1891, Lieut.-Commander Z. L. Tanner, U.S.N., commanding, 15 . The stalk-eyed Crustacea. Mem. Mus. comp. Zool. Harvard, $18: 1-292$, figs. $1-6$, pls. A-K + 1-57.

Forest, J., 1955. Crustacés Décapodes, Pagurides. Rés. Sci. Expéd. océanogr. belge eaux côtières afr. Atlantique sud (1948 - 1949), 3 (4) : 21-147, pls. 1-6.

-, 1961. Pagurides de l'Afrique occidentale. Atlantide Rep., 6 : 203-250, figs. $1-19$.

Forest, J. \& M. de Saint Laurent, 1968. Campagne de la "Calypso" au large des côtes atlantiques de l'Amérique du sud (1961-1962), (Première partie), 6. Crustacés Décapodes: Pagurides. Rés. sci. Camp. Calypso, 8. Ann. Inst. océanogr., 45 (2), 1967 (1968),: 47-169, figs. $1-148$, pl. 1.

Hale, H., 1941. Decapod Crustacea. Rep. B.A.N.Z. Antarctic Research Exped. 1929-1931 (B) (Zool. Bot.), 4 (9) : 257-285, figs. $1-16$.

Henderson, J. R., 1885. In : T. H. Tizard et al., Narrative of the cruise of H.M.S. Challenger with a general account of the scientific results of the expedition. Sci. Rep. Challenger Exped., 1 (2) : 5111110 , figs. $1-340$.

-, 1888. Report on the Anomura collected by H.M.S. Challenger during the years 1873-76. Sci. Rep. Challenger Exped. (Zool.), 27: i-xi + 1-221, pls. $1-21$.

-, 1896. Report on the Paguridae collected during the season 1893-94. Natural history notes from H.M. Indian Marine Survey Steamer "Investigator", Commander C. F. Oldham, R.N., commanding, (2) 24. J. Asiat. Soc. Bengal, 15 (2) : 516-536.

Holmes, S. J., 1900. Synopsis of California stalk-eyed Crustacea. Occ. Pap. Calif. Acad. Sci., 7 : 1-162, pls. $1-4$.

MacDonald, J. D., R. B. Pike \& D. I. Williamson, 1957. Larvae of the British species of Diogenes, Pagurus, Anapagurus and Lithodes (Crustacea Decapoda). Proc. zool. Soc. London, 128 : 209-257, figs. $1-11$.

Makarov, V. V., 1938. Anomura. Fauna SSSR, Rakoobrazyne, 10 (3) : $i-x+1-324$, figs. $1-113$, pls. 1-5. (Akad. Nauk SSSR, Zool. Inst., n. Sér., 16).

Milne EdWards, A., 1880. Reports on the results of dredging under the supervision of Alexander Agassiz, in the Gulf of Mexico, and in the Caribbean Sea,
1877, '78, '79, by the U.S. Coast Survey Steamer "Blake", Lieut.-Commander C. D. Sigsbee, U.S.N., and Commander J. R. Bartlett, U.S.N., Commanding, 8. Etudes préliminaires sur les Crustacés, 1. Bull. Mus. comp. Zool. Harvard, 8 ; 1-68, pls. 1-2. -, 1884. L'expédition du "Talisman". Bull. hebd. Assoc. Sci. France, (2) 8, 1883 (1884) : 157-179.

-, 1891. Pagurides nouveaux des Açores. Campagnes scientifiques de S.A. le Prince de Monaco sur le yacht 1"'Hirondelle". Bull. Soc. zool. France, 16: 131-134.

Milne Edwards, A. \& E.-L. Bouvier, 1893. Reports on the results of dredging, under the supervision of Alexander Agassiz, in the Gulf of Mexico (187778), in the Caribbean Sea (1878-79), and along the atlantic coast of the United States (1880), by the U.S. Coast Survey Steamer "Blake", Lieut.-Comm. S. D. Sigsbee, U.S.N., and Commander J. R. Bartlett, U.S.N., commanding, 33. Description des Crustacés de la famille des Paguriens recueillis pendant l'expédition. Mem. Mus. comp. Zool. Harvard, 14 (3) : $1-172$, pls. $1-12$.

- \& -, 1894a. Crustacés Décapodes provenant des campagnes du yacht l"'Hirondelle" (1886, 1887 et 1888). I. Brachyures et Anomoures. Rés. Camp. sci. Monaco, 7 : $1-112$, figs. $A-D$, pls. $1-11$.

- \& -, 1894 b. Considérations générales sur la famille des Galathéides. Ann. Sci. nat., (Zool.) (7) $16: 191$ 327 , figs. $1-35$.

— \& -, 1897. Observations sur le genre Sympagurus. Campagnes scientifiques de S. A. le Prince Albert ler de Monaco à bord de 1"'Hirondelle" et de la "Princesse Alice". Bull. Soc. zool. France, 22 : $131-136$.

— \& -, 1899. Crustacés Décapodes provenant des campagnes de 1"'Hirondelle" (supplément) et de la "Princesse Alice" (1891-1897). Rés. Camp. sci. Monaco, 13 : $1-106$, pls. $1-4$.

— \& -, 1900. Crustacés Décapodes, 1. Brachyures et Anomoures. Expéditions scientifiques du "Travailleur" et du "Talisman" pendant les années 1880 , 1881,1882 et $1883: 1-396$, pls. 1-32. (Paris).

Ortmann, A., 1922. Die Decapoden-Krebse des Strassburger Museums, 4. Die Abtheilungen Galatheidea und Paguridea. Zool. Jahrb. (Syst.), 6: 241-236, pls. $11-12$.

Saint LaURent-Dechancé, M. De, 1964. Développement et position systématique du genre Parapagurus Smith (Crustacea Decapoda Paguridea), 1. Description des stades larvaires. Bull. Inst. océanogr. Monaco, 64 (1321) : $1-26$, figs. $1-23$.

-, 1966. Remarques sur la classification des Paguridae et sur la position systématique d'Iridopagurus de Saint Laurent. Diagnose d'Anapagrides gen. nov. Bull. Mus. nation. Hist. nat., Paris, (2) 38 (3) : 257 $-265$.

Saint Laurent, M. De, 1968. Révision des genres Catapaguroides et Cestopagurus et description de quatre genres nouveaux, 1. Catapaguroides A. Milne Edwards et Bouvier et Decaphyllus nov. gen. (Crustacés Décapodes Paguridae). Bull. Mus. nation. Hist. nat., Paris, (2) 39 (5-6) 1967 (1968) : 923—954, 1100 1119, figs. $1-56$. 
- 1970. Révision des genres Catapaguroides ot Cestopagurus et description de quatre genres nouveaux, 5 . Trichopagurus de Saint Laurent (Crustacés Décapodes Paguridae). 6. Conclusion. Bull. Mus. nation. Hist. nat., Paris, (2) 42 (1) : $210-222$, figs. $1-16$.

SmITH, S. I., 1879. The stalk-eyed crustaceans of the Atlantic Coast of North America North of Cape Cod. Trans. Conn. Acad. Arts Sci., 5: 27-136, pls. 8-12.

-, 1882. Reports on the results of dredgings, under the supervision of Alexander Agassiz, on the East coast of the United States, during the summer of 1880 , by the U.S. Coast Survey Steamer "Blake" Commander J. R. Bartlett, U.S.N., commanding, 17. Report on the Crustacea, 1 : Decapoda. Bull. Mus. comp. Zool. Harvard, 10 (1) : 1-108, pls. 1-16.

- , 1883. Preliminary report on the Brachyura and Anomura dredged in deep water off the South coast of New England by the U.S. Fish Commission in 1880, 1881 and 1882. Proc. U.S. nation. Mus., 6: $1-57$, pls. $1-6$.

- , 1884. Report on the decapod Crustacea of the "Albatross" dredgings off the East coast of the United States in 1883. Ann. Rep. Comm. Fish Fish. 1882 : $345-424$, pls. $1-10$.

-, 1886. Report on the decapod Crustacea of the "Albatross" dredgings off the East coast of the United States during the summer and autumn of 1884. Ann. Rep. Comm. Fish Fish. 1885 : 605-706, pls. 1-20.

Stebbing, T. R. R., 1910. General catalogue of South African Crustacea. Ann. S. Afr. Mus., 6 : 281-599, pls. $15-22$.
STUDER, T., 1883. Verzeichniss der Crustaceen, welche während der Reise S.M.S. Gazelle an der Westküste von Afrika, Ascension und dem Cap der guten Hoffnung gesammelt wurden. Abh. preuss. Akad. Wiss., phys.-math. Kl., 2 (1882-1883): 1-32, pls. 1-2.

Terao, A., 1913. A catalogue of Hermit-crabs found in Japan (Paguridea excluding Lithodidae), with description of four new species. Annot. zool. jap., 8 (2) : 355-391, figs. $1-4$.

Thompson, E. F., 1943. Paguridae and Coenobitidae. Sci. Rep. John Murray Exped. 1933-34, 7 (5) : 411 -426 , figs. $1-2$.

Whitelegge, T., 1900. Crustacea, 1. Sci. Res. "Thetis". Mem. Aust. Mus., 4: $135-199$, figs. $11-14$, pls. 32-35.

Williamson, D. I. \& K. G. von Levetzow, 1967. Larvae of Parapagurus diogenes (Whitelegge) and some related species (Decapoda, Anomura). Crustaceana, 12 (2) : 179-192, figs. 1-3.

WolfF, T., 1960. Strejflys over dybhavets dyreliv. Naturens Verden, Copenhague, Juni 1960 : 161-191.

-, 1961. Description of a remarkable deep-sea hermitcrab, with notes on the evolution of the Paguridae. Galathea Rep., 4: 11-32, figs. 1-11.

YoxoyA, Y., 1933. On the distribution of decapod crustaceans inhabiting the continental shelf around Japan, chiefly based upon the materials collected by S.S. Sôyô-Maru, during the years 1923-1930. J. agric. Coll. Tokyo, 12 (1) : $1-226$, figs. $1-71$. 